\title{
A Probabilistic Separation Logic
}

GILLES BARTHE, MPI for Security and Privacy, Germany and IMDEA Software Institute, Spain

JUSTIN HSU, University of Wisconsin-Madison, USA

KEVIN LIAO, MPI for Security and Privacy, Germany and University of Illinois Urbana-Champaign, USA

Probabilistic independence is a useful concept for describing the result of random sampling-a basic operation in all probabilistic languages-and for reasoning about groups of random variables. Nevertheless, existing verification methods handle independence poorly, if at all. We propose a probabilistic separation logic PSL, where separation models probabilistic independence. We first give a new, probabilistic model of the logic of bunched implications (BI). We then build a program logic based on these assertions, and prove soundness of the proof system. We demonstrate our logic by verifying information-theoretic security of cryptographic constructions for several well-known tasks, including private information retrieval, oblivious transfer, secure multi-party addition, and simple oblivious RAM. Our proofs reason purely in terms of high-level properties, like independence and uniformity.

CCS Concepts: • Security and privacy $\rightarrow$ Logic and verification; $\bullet$ Theory of computation $\rightarrow$ Separation logic.

Additional Key Words and Phrases: probabilistic independence, separation logic, verified cryptography

ACM Reference Format:

Gilles Barthe, Justin Hsu, and Kevin Liao. 2020. A Probabilistic Separation Logic. Proc. ACM Program. Lang. 4, POPL, Article 55 (January 2020), 30 pages. https://doi.org/10.1145/3371123

\section{INTRODUCTION}

Probabilistic programs have important applications in many domains, including information security and machine learning. As the impact of these areas continues to grow, probabilistic programming languages (PPLs) are receiving renewed attention from formal verification. While the mathematical semantics of PPLs has been well-studied, starting from Kozen [1981]; Saheb-Djahromi [1980] and continuing up to today [Ehrhard et al. 2018; Vákár et al. 2019], deductive program verification for PPLs remains challenging. Establishing simple properties can involve tedious arguments, and scaling formal proofs up to verify target properties of randomized algorithms is often difficult.

\subsection{Probabilistic Independence}

A basic property that is poorly handled by existing verification techniques is independence. Roughly speaking, two random variables are probabilistically independent if they are uncorrelated: information about one quantity yields no information about the other. In probabilistic programs, independence usually arises when variables are derived from separate randomness-e.g., from the results of two different coin flips-but independence can also hold when variables share randomness in just the right way.

Authors' addresses: Gilles Barthe, MPI for Security and Privacy, Germany and IMDEA Software Institute, Spain; Justin Hsu, University of Wisconsin-Madison, USA; Kevin Liao, MPI for Security and Privacy, Germany and University of Illinois Urbana-Champaign, USA.

This work is licensed under a Creative Commons Attribution 4.0 International License.

(C) 2020 Copyright held by the owner/author(s).

2475-1421/2020/1-ART55

https://doi.org/10.1145/3371123

Proc. ACM Program. Lang., Vol. 4, No. POPL, Article 55. Publication date: January 2020. 
Although it is usually not the target property of interest, probabilistic independence often serves as an intermediate assertion in pen-and-paper proofs of randomized algorithms. From a verification perspective, independence is useful for several reasons.

Independence simplifies reasoning about groups of random variables. Probabilistic programs often manipulate multiple random variables. If a group of random variables are independent, then their joint distribution is precisely described by the distribution of each variable in isolation. As a result, formal reasoning can focus on one variable at a time, without losing information.

Independence characterizes the result of random sampling. All PPLs have built-in constructs to draw random samples from primitive distributions (e.g., drawing a random boolean from a coin-flip distribution). These basic operations produce a "fresh" random quantity that is independent from the rest of the program state, at least when the primitive distribution does not depend on the state

Independence is preserved under local operations. Like standard programs, probabilistic programs typically manipulate only a few variables at a time. To ease formal reasoning, properties about unmodified variables should be preserved as much as possible. Independence is preserved under local modifications: if $x$ and $y$ are independent and $x$ is updated to $x^{\prime}=x+1$, then $x^{\prime}$ and $y$ remain independent. In this way, probabilistic independence seemingly flows through a program, continuing to hold far beyond the original sampling instructions.

Independence is compatible with conditioning. Probabilistic programs can have randomized control flow, for example branching on a randomized boolean. Semantically, this kind of branch is modeled by conditioning, an operation that transforms an input distribution into two conditional distributions, one where the guard is true and one where the guard is false.

While conditioning is well-understood mathematically, it poses problems for formal reasoning. Conditioning on a variable $x$-say, when branching on $x>0$-can alter the distribution over other variables. If $x$ is independent of $y$, however, conditioning on $x$ will have no effect on the distribution over $y$. As a result, properties of variables that are syntactically separate from the variable $x$ are preserved when conditioning on the guard $x>0$, a highly useful reasoning principle.

\subsection{Example Applications of Independence}

To ground our investigation in applications, we focus on security properties from cryptography. We first encode target cryptographic protocols as probabilistic programs of type $\mathcal{A} \times \mathcal{A} \rightarrow O \times \mathcal{A}$, where the first input represents the secret input, the second input represents the public input, and the first and second output represents the observer's view and computation output, respectively. In many cases the two outputs coincide, but this need not be the case in general.

Then, we establish security properties by proving properties of these programs in our logic. Defining precisely what it means for a construction to be secure is surprisingly subtle; cryptographers have proposed many definitions capturing different assumptions and guarantees. Baseline, information-theoretic security of many schemes, including private information retrieval [Chor et al. 1995], oblivious transfer [Rivest 1999], multi-party computation [Cramer et al. 2015], and oblivious RAM [Chung and Pass 2013], can be stated in terms of the following definitions.

Uniformity. A natural way to define security is to require that the observer's view is the same, no matter what the private input is; this is a probabilistic form of non-interference. For instance, it suffices to show that the observer's view is always uniformly distributed over a fixed set: no matter what the private inputs are, the observer's view is the same.

Input Independence. Another way to define security is to model the secret input as drawn from some distribution, and then argue that the distribution of the observer's view is probabilistically 
independent of the secret input. This formulation captures security through an intuitive reading of independence: the observer's view reveals no information about the secret input. Though this definition looks quite different from probabilistic non-interference, the two definitions are equivalent in many settings. Their proofs, however, may be quite different.

\subsection{Contributions and Plan of the Paper}

After introducing mathematical preliminaries in Section 2, we begin working towards the main goal of this paper: a probabilistic program logic where independence is the central concept. Our logic is a probabilistic variant of separation logic, a highly successful technique for reasoning about heap-manipulating programs [Ishtiaq and O'Hearn 2001; O'Hearn et al. 2001]. To model sharing and separation, separation logic uses assertions from the logic of bunched implications (BI), a substructural logic. For instance, the separating conjunction models separation of heaps: $\phi * \psi$ states that the heap can be split into two disjoint parts satisfying $\phi$ and $\psi$, respectively.

While separation logic was originally designed for heaps, separation is a useful concept in many verification settings. A notable line of work extends separation logic to the concurrent setting, where separation models exclusive ownership of resources [Brookes 2007; O'Hearn 2007]. More generally, the resource semantics of BI [Pym et al. 2004] gives a powerful way to generalize BI to new notions of separation.

Inspired by this perspective, our first contribution is a new interpretation of BI where the separating conjunction models probabilistic independence. Roughly speaking, $\phi * \psi$ holds in a distribution $\mu$ over program memories if $\mu$ can be factored into two distributions $\mu_{1}$ and $\mu_{2}$ satisfying $\phi$ and $\psi$, respectively. Splitting a distribution amounts to finding two disjoint sets of program variables $X$ and $Y$ such that every distribution in the support of $\mu$ is defined precisely on $X \cup Y$, with the factors $\mu_{1}$ and $\mu_{2}$ obtained by projecting $\mu$ along $X$ and $Y$ respectively. This intuitive interpretation gives rise to a probabilistic model of BI. Our model can smoothly incorporate useful primitive assertions about distributions, including probabilistic equality and uniformity. We present our model in Section 3.

Leveraging this probabilistic version of $\mathrm{BI}$ as an assertion logic, our second contribution is a program logic PSL for a simple probabilistic programming language, similar to PWHILE. Our logic bears a strong resemblance to separation logic: there are proof rules for local and global reasoning, there is a version of the Frame rule, and whereas separation logic distinguishes between store and heap, our logic distinguishes between deterministic and probabilistic variables. However, there are also notable differences in the probabilistic setting. We present the proof system of PSL and prove soundness in Section 4.

As our third contribution, we demonstrate our program logic by formalizing security of several well-known constructions from cryptography, including a simple oblivious RAM, a private information retrieval algorithm, a simple three-party computation algorithm for addition, and an oblivious transfer algorithm. We prove two different forms of information-theoretic security: uniformity of outputs (which implies probabilistic non-interference), and input independence. We present these examples in Section 5.

We survey related work in Section 6 and discuss potential future directions in Section 7.

\section{PRELIMINARIES}

\subsection{Probabilities and Distributions}

A (discrete) probability distribution over a countable set $A$ is a function $\mu: A \rightarrow[0,1]$ such that the total weight is one: $\sum_{a \in A} \mu(a)=1$; we write $\mathbf{D}(A)$ for the set of all distributions over $A$. Intuitively, $\mu(a)$ represents the probability of drawing $a$ from the distribution $\mu$. Likewise, the probability of 
drawing some element in $S \subseteq A$ is $\mu(S) \triangleq \sum_{a \in S} \mu(a)$. The support of a distribution is the set of elements with non-zero probability: $\operatorname{supp}(\mu)=\{a \in A \mid \mu(a)>0\}$.

We will use two standard constructions on probability distributions. First, the distribution unit unit : $A \rightarrow \mathrm{D}(A)$ associates each element $a \in A$ with the Dirac distribution $\delta_{a}$ centered at $a$. This distribution is simply defined as $\delta_{a}(x) \triangleq 1$ if $x=a$ and $\delta_{a}(x) \triangleq 0$ otherwise; intuitively, the Dirac distribution deterministically yields $a$. Second, the distribution bind bind $: \mathbf{D}(A) \rightarrow(A \rightarrow \mathbf{D}(B)) \rightarrow$ $\mathbf{D}(B)$ is defined by:

$$
\operatorname{bind}(\mu, f)(b) \triangleq \sum_{a \in A} \mu(a) \cdot f(a)(b)
$$

Intuitively, bind sequences a distribution with a continuation. Together, unit and bind make $\mathrm{D}$ a monad [Giry 1982]; these operations are commonly used to model randomized programs.

Given our focus on independence, we will be particularly interested in distributions over products and products of distributions. The distribution product $\otimes: \mathbf{D}(A) \times \mathbf{D}(B) \rightarrow \mathbf{D}(A \times B)$ is defined by:

$$
\left(\mu_{A} \otimes \mu_{B}\right)(a, b) \triangleq \mu_{A}(a) \cdot \mu_{B}(b) .
$$

We can extract component distributions out of any distribution over a product using the projections $\pi_{1}: \mathbf{D}(A \times B) \rightarrow \mathbf{D}(A)$ and $\pi_{2}: \mathbf{D}(A \times B) \rightarrow \mathbf{D}(B):$

$$
\pi_{1}(\mu)(a) \triangleq \sum_{b \in B} \mu(a, b) \quad \text { and } \quad \pi_{2}(\mu)(b) \triangleq \sum_{a \in A} \mu(a, b) .
$$

We call $\mu \in \mathbf{D}(A \times B)$ a product distribution if it can be factored as $\mu=\pi_{1}(\mu) \otimes \pi_{2}(\mu)$; in this case, we say that the components of $\mu$ are (probabilistically) independent.

Finally, will need conditioning and convex combination operations on distributions to model control flow splits and merges, respectively. Let $S \subseteq A$ be any event. If $S$ has non-zero probability under $\mu \in \mathbf{D}(A)$, then the conditional distribution $\mu \mid S \in \mathbf{D}(A)$ is defined as:

$$
(\mu \mid S)(E) \triangleq \frac{\mu(S \cap E)}{\mu(S)}
$$

Intuitively, the conditional distribution represents the relative probabilities of elements restricted to $S$. Conditioning is not defined when $\mu(S)=0$.

To join output distributions from two branches, we define the convex combination of distributions. Let $\rho \in[0,1]$ and let $\mu_{1}, \mu_{2} \in \mathbf{D}(A)$. The convex combination $\mu_{1} \oplus_{\rho} \mu_{2} \in \mathbf{D}(A)$ is defined as:

$$
\left(\mu_{1} \oplus_{\rho} \mu_{2}\right)(S) \triangleq \rho \cdot \mu_{1}(S)+(1-\rho) \cdot \mu_{2}(S)
$$

We define $\mu_{1} \oplus_{0} \mu_{2} \triangleq \mu_{2}$ and $\mu_{1} \oplus_{1} \mu_{2} \triangleq \mu_{1}$, even when $\mu_{1}$ or $\mu_{2}$ may be undefined. Conditioning and taking convex combination yields the original distribution: $\mu=(\mu \mid S) \oplus_{\mu(S)}(\mu \mid \bar{S})$.

\subsection{Probabilistic Memories}

Distributions over program memories are naturally modeled by distributions over products. We fix a countable set $\mathcal{R} \mathcal{V}$ of random variables and a countable set Val of values. For any subset of variables $S \subseteq \mathcal{R} \mathcal{V}$, we let $\operatorname{RanM}[S] \triangleq S \rightarrow$ Val be the set of memories with domain $S$; we write $\operatorname{RanM} \triangleq \operatorname{RanM}[\mathcal{R} \mathcal{V}]$. When $S$ is empty, there is precisely one map $0: \emptyset \rightarrow$ Val and so $\mathrm{D}(\operatorname{RanM}[\emptyset])$ contains just the Dirac distribution $\delta_{0}$. Given a distribution $\mu \in \mathbf{D}(\operatorname{RanM}[S])$, we write $\operatorname{dom}(\mu) \triangleq S$ for the domain.

Viewing $\operatorname{RanM}[S]$ as a product indexed by $S$, we can adapt the general constructions for distributions over products to distributions over $\operatorname{RanM}[S]$. Given disjoint variables $S, S^{\prime} \subseteq \mathcal{R} \mathcal{V}$, for instance, we define the product $\otimes: \mathbf{D}(\operatorname{RanM}[S]) \times \mathbf{D}\left(\operatorname{RanM}\left[S^{\prime}\right]\right) \rightarrow \mathbf{D}\left(\operatorname{RanM}\left[S \cup S^{\prime}\right]\right)$ to be

$$
\left(\mu_{S} \otimes \mu_{S^{\prime}}\right)(m) \triangleq \mu_{S}\left(m_{S}\right) \cdot \mu_{S^{\prime}}\left(m_{S^{\prime}}\right)
$$


where $m_{S} \in \operatorname{RanM}[S]$ and $m_{S^{\prime}} \in \operatorname{RanM}\left[S^{\prime}\right]$ restrict $m$ to $S$ and $S^{\prime}$ respectively. The Dirac distribution $\delta_{0}$ is the identity of this operation: $\mu \otimes \delta_{0}=\delta_{0} \otimes \mu=\mu$. When $\mu \in \mathrm{D}(\operatorname{RanM}[S])$ can be factored as $\mu=\mu_{1} \otimes \mu_{2}$ for $\mu_{i} \in \mathbf{D}\left(\operatorname{RanM}\left[S_{i}\right]\right)$, we say that $S_{1}$ and $S_{2}$ are (probabilistically) independent in $\mu$.

Likewise, we can project a distribution over $\operatorname{RanM}[S]$ to a distribution over $\operatorname{RanM}\left[S^{\prime}\right]$ for $S^{\prime} \subseteq S$ using the projection $\pi_{S, S^{\prime}}: \mathbf{D}(\operatorname{RanM}[S]) \rightarrow \mathbf{D}\left(\operatorname{RanM}\left[S^{\prime}\right]\right)$, defined as:

$$
\pi_{S, S^{\prime}}(\mu)\left(m_{S^{\prime}}\right) \triangleq \sum_{m_{S} \in \operatorname{RanM}[S]: p_{S^{\prime}}\left(m_{S}\right)=m_{S^{\prime}}} \mu\left(m_{S}\right),
$$

where $p_{S^{\prime}}$ restricts the range of the memory to $S^{\prime}$. For example, $\pi_{S, S}$ is the identity, while $\pi_{S, \emptyset}$ maps all distributions to $\delta_{0}$. We will abbreviate $\pi_{S, S^{\prime}}$ by $\pi_{S^{\prime}}$ (or just $\pi$ ) when the domains are clear from the context.

\subsection{Probabilistic Programs}

We will work with a variant of the basic probabilistic imperative language PWHILE. We enforce a clear separation between deterministic and probabilistic data using simple syntactic conditions, though more sophisticated techniques (e.g., dataflow analysis) could be also used. Let $\mathcal{D} \mathcal{V}$ be a countable set of deterministic variables disjoint from $\mathcal{R} \mathcal{V}$, and let $\operatorname{Det} \mathrm{M} \triangleq \mathcal{D} \mathcal{V} \rightarrow$ Val be the set of deterministic memories, or stores. The expression language is largely standard:

$$
\begin{aligned}
\mathcal{D E} \ni e_{d}::=\mathcal{D V}|\mathcal{D E}+\mathcal{D E}| \mathcal{D E} \wedge \mathcal{D E} \mid \cdots \\
\mathcal{R E} \ni e_{r}::=\mathcal{D E}|\mathcal{R} \mathcal{V}| \mathcal{R} \mathcal{E}+\mathcal{R} \mathcal{E}|\mathcal{R} \mathcal{E} \wedge \mathcal{R} \mathcal{E}| \cdots
\end{aligned}
$$

We assume that expressions are typed using a simple type system, and we only work with welltyped expressions. We interpret deterministic expressions as maps $\llbracket e_{d} \rrbracket:$ DetM $\rightarrow$ Val. Randomized expressions are interpreted as maps $\llbracket e_{r} \rrbracket: \operatorname{Det} \mathrm{M} \times \operatorname{RanM}[S] \rightarrow$ Val, where $S \subseteq \mathcal{R} \mathcal{V}$ contains all randomized variables in $e_{r}$; if $e_{r}$ mentions variables outside of $S$, then the interpretation is not defined. It is also straightforward to lift this interpretation to interpret randomized expressions in distributions over randomized memories: $\llbracket e_{r} \rrbracket: \operatorname{Det} \mathbf{M} \times \mathbf{D}(\operatorname{RanM}[S]) \rightarrow \mathbf{D}(\operatorname{Val})$.

Next, we consider the commands. $\mathcal{R} C$ commands appearing under a randomized guard-so they cannot assign to deterministic variables-while $C$ commands are general.

$$
\begin{aligned}
& \mathcal{R} C \ni c::=\operatorname{skip}|\mathcal{R} \mathcal{V} \leftarrow \mathcal{R E}| \mathcal{R V} \leftrightarrow \mathrm{U}_{S} \mid \mathcal{R} C ; \mathcal{R} C \\
& \text { | } \text { if }_{D} \mathcal{D E} \text { then } \mathcal{R} C \text { else } \mathcal{R} C \mid \text { if }_{R} \mathcal{R E} \text { then } \mathcal{R} C \text { else } \mathcal{R} C \mid \text { while } \mathcal{D E} \text { do } \mathcal{R} C \\
& C \ni c::=\operatorname{skip}|\mathcal{D V} \leftarrow \mathcal{D} \mathcal{E}| \mathcal{R V} \leftarrow \mathcal{R} \mathcal{\mathcal { V }}\left|\mathcal{R} \mathcal{V} \leftarrow \mathrm{U}_{S}\right| C ; C \\
& \text { | if }{ }_{D} \mathcal{D E} \text { then } C \text { else } C \mid \text { if }_{R} \mathcal{R E} \text { then } \mathcal{R} C \text { else } \mathcal{R} C \mid \text { while } \mathcal{D E} \text { do } C
\end{aligned}
$$

The main probabilistic command is sampling: $\mathcal{R} \mathcal{V} \leftrightarrow \mathrm{U}_{S}$ takes a uniform sample from a finite, non-empty set $S$ and assigns it to a variable.

Our grammar separates commands for assignments to deterministic variables and randomized variables, and separates commands for deterministic and probabilistic conditionals (we require loop guards to be deterministic). These distinctions will be important when we introduce our proof system. We will also use a few standard variants of commands:

$$
\begin{gathered}
\text { for } i=1, \ldots, N \text { do } c \triangleq i \leftarrow 1 ; \text { while } i \leq N \text { do }(c ; i \leftarrow i+1) \\
\text { if }_{D} b \text { then } c \triangleq \text { if }_{D} b \text { then } c \text { else skip } \quad \text { if }_{R} b \text { then } c \triangleq \text { if }_{R} b \text { then } c \text { else skip }
\end{gathered}
$$

We interpret programs using a restricted version of the standard semantics due to Kozen [1981], assuming additionally that programs terminate on all inputs-notions like product distribution and probabilistic independence are poorly behaved when programs may diverge with positive 


$$
\begin{aligned}
& \llbracket \text { skip } \rrbracket(\sigma, \mu) \triangleq(\sigma, \mu) \\
& \llbracket x_{d} \leftarrow e_{d} \rrbracket(\sigma, \mu) \triangleq\left(\sigma\left[x_{d} \mapsto \llbracket e_{d} \rrbracket \sigma\right], \mu\right) \\
& \llbracket x_{r} \leftarrow e_{r} \rrbracket(\sigma, \mu) \triangleq\left(\sigma, \operatorname{bind}\left(\mu, m \mapsto \operatorname{unit}\left(m\left[x_{r} \mapsto \llbracket e_{r} \rrbracket(\sigma, m)\right]\right)\right)\right) \\
& \llbracket x_{r} \leftrightarrow \mathbf{U}_{S} \rrbracket(\sigma, \mu) \triangleq\left(\sigma, \operatorname{bind}\left(\mu, m \mapsto \operatorname{bind}\left(\operatorname{Unif}_{S}, u \mapsto \operatorname{unit}\left(m\left[x_{r} \mapsto u\right]\right)\right)\right)\right) \\
& \llbracket c ; c^{\prime} \rrbracket(\sigma, \mu) \triangleq \llbracket c^{\prime} \rrbracket(\llbracket c \rrbracket(\sigma, \mu)) \\
& \llbracket \text { if }_{D} b \text { then } c \text { else } c^{\prime} \rrbracket(\sigma, \mu) \triangleq \begin{cases}\llbracket c \rrbracket(\sigma, \mu) & : \llbracket b \rrbracket \sigma=\text { true } \\
\llbracket c^{\prime} \rrbracket(\sigma, \mu) & : \llbracket b \rrbracket \sigma=\text { false }\end{cases} \\
& \llbracket \text { if }_{R} b \text { then } c \text { else } c^{\prime} \rrbracket(\sigma, \mu) \triangleq \llbracket c \rrbracket(\sigma, \mu \mid \llbracket b \rrbracket \sigma=\text { true }) \oplus_{\rho} \llbracket c^{\prime} \rrbracket(\sigma, \mu \mid \llbracket b \rrbracket \sigma=\text { false }) \\
& \text { where } \rho=\mu(\llbracket b \rrbracket \sigma=\text { true }) \\
& \llbracket \text { while } b \text { do } c \rrbracket(\sigma, \mu) \triangleq \llbracket \underbrace{c ; \cdots ; c} \rrbracket(\sigma, \mu) \\
& N(\sigma) \triangleq \text { \#steps until } \llbracket b \rrbracket=\text { false }
\end{aligned}
$$

Fig. 1. Program semantics

probability. Technically, programs transform configurations, pairs of a deterministic memory $\sigma$ and a distribution $\mu$ over randomized memories:

$$
\llbracket c \rrbracket:(\operatorname{DetM} \times \mathbf{D}(\operatorname{RanM})) \rightarrow(\operatorname{Det} M \times \mathbf{D}(\operatorname{RanM})) .
$$

Figure 1 presents the program semantics; $\operatorname{Unif}_{S} \in \mathbf{D}(S)$ is the uniform distribution over a finite, non-empty set $S$, which assigns probability $1 /|S|$ to every element $s \in S$. The semantics of random conditionals uses convex combination lifted to configurations; this is defined since the output stores are equal because branches under random guards may not modify deterministic variables.

\section{A PROBABILISTIC MODEL OF BI}

Assertions in separation logic are based on the logic of bunched implications (BI) [O'Hearn and Pym 1999; Pym 1999]. We first review the syntax and semantics of this logic, then introduce a probabilistic interpretation that will enable BI formulas to describe probabilistic states.

\subsection{The Syntax and (Resource) Semantics of BI, in Brief}

The logic of bunched implications (BI) is a substructural logic with the following formulas: ${ }^{1}$

$$
\phi, \psi::=p \in \mathcal{A P}|\top| \perp|\phi \wedge \psi| \phi \vee \psi|\phi \rightarrow \psi| \phi * \psi \mid \phi * \psi
$$

Throughout, $p$ ranges over a set of atomic propositions $\mathcal{A P}$. Negation $\neg \phi$ is defined as $\phi \rightarrow \perp$. Intuitively, BI combines standard propositional logic with a substructural fragment consisting of the separating conjunction * and the separating implication ("magic wand") $*$. In the standard heap model of BI underlying separation logic, atomic propositions describe the contents of particular heap locations, separating conjunction combines assertions describing disjoint portions of the heap, and separating implication describes the result of adjoining the current heap with a disjoint portion.

BI can be given several kinds of semantics. We follow the resource semantics, as developed by David Pym and others [Pym et al. 2004]. The basic idea is to define a Kripke semantics where the set of possible worlds forms a partial, pre-ordered commutative monoid $\mathcal{M}=(M, \circ, e, \sqsubseteq)$.

\footnotetext{
${ }^{1}$ We do not need the multiplicative identity $I$ from BI, since it will be equivalent to $\mathrm{T}$ in our setting.
} 
Definition 1 (Galmiche et al. [2005]). A (partial) Kripke resource monoid consists of a set $M$ of possible worlds, a partial binary operation $\circ: M \times M \rightarrow M$, an element $e \in M$, and a pre-order $\sqsubseteq$ on $M$ such that the monoid operation

- has identity $e$ : for all $x \in M$, we have $e \circ x=x \circ e=x$;

- is associative: $x \circ(y \circ z)=(x \circ y) \circ x$, where both sides are either defined and equal, or both undefined; and

- is compatible with the pre-order: if $x \sqsubseteq y$ and $x^{\prime} \sqsubseteq y^{\prime}$ and if both $x \circ x^{\prime}$ and $y \circ y^{\prime}$ are defined, then $x \circ x^{\prime} \sqsubseteq y \circ y^{\prime}$.

Under the resource interpretation of BI, possible worlds are collections of resources, the monoid operation $\circ$ combines sets of resources, and the identity $e$ represents the lack of resources. The monoid operation may fail to be defined when combining two incompatible sets of resources; this is useful for modeling resources that should not be duplicated, such as heap addresses. We write $\left(m \circ m^{\prime}\right) \downarrow$ when the combination is defined.

Definition 2. Let $(M, \circ, e, \sqsubseteq)$ be a partial Kripke resource monoid and let $\llbracket-\rrbracket: \mathcal{A P} \rightarrow 2^{M}$ be a Kripke resource interpretation of atomic formulas: if $m \in \llbracket p \rrbracket$ and $m \sqsubseteq m^{\prime}$, then $m^{\prime} \in \llbracket p \rrbracket$. Then the corresponding Kripke resource model of BI can be defined as follows:

$$
\begin{aligned}
& m \vDash p \quad \text { iff } m \in \llbracket p \rrbracket \\
& m \vDash T \quad \text { always } \\
& m \vDash \perp \quad \text { never } \\
& m \vDash \phi \wedge \psi \quad \text { iff } m \vDash \phi \text { and } m \vDash \psi \\
& m \vDash \phi \vee \psi \quad \text { iff } m \vDash \phi \text { or } m \vDash \psi \\
& m \vDash \phi \rightarrow \psi \quad \text { iff for all } m \sqsubseteq m^{\prime}, m^{\prime} \vDash \phi \text { implies } m^{\prime} \vDash \psi \\
& m \vDash \phi * \psi \quad \text { iff exist } m_{1}, m_{2} \text { with }\left(m_{1} \circ m_{2}\right) \downarrow \text { and } m_{1} \circ m_{2} \sqsubseteq m \text { such that } m_{1} \vDash \phi \text { and } m_{2} \vDash \psi \\
& m \vDash \phi * \psi \quad \text { iff for all } m^{\prime} \text { such that } m^{\prime} \vDash \phi,\left(m \circ m^{\prime}\right) \downarrow \text { implies } m \circ m^{\prime} \vDash \psi
\end{aligned}
$$

All formulas satisfy the Kripke monotonicity property: if $m \models \phi$ and $m \sqsubseteq m^{\prime}$, then $m^{\prime} \vDash \phi$ as well. We write $=\phi$ when $\phi$ is valid, i.e., when $\phi$ holds in all worlds.

$\mathrm{BI}$ enjoys good metatheoretic properties and a rich proof theory. Many models are known beyond heaps, including doubly closed categories (DCCs), presheafs, and Petri nets. There are also complete proof systems for BI. The interested reader should consult Pym [2002] or Docherty [2019] for a detailed treatment of BI's proof theory, and Galmiche et al. [2005] for more information about the partial monoid semantics we use here.

\subsection{A Probabilistic Version of BI}

By leveraging the resource semantics of BI, we can give a probabilistic interpretation of BI formulas.

Definition 3. Let $M$ be the set of program configurations $\operatorname{Det} \mathrm{M}[S] \times \mathrm{D}(\operatorname{RanM}[T])$ where $S$ ranges over subsets of $\mathcal{D} \mathcal{V}$ and $T$ ranges over subsets of $\mathcal{R} \mathcal{V}$. Let $\circ$ be a partial binary operation defined as:

$$
(\sigma, \mu) \circ\left(\sigma^{\prime}, \mu^{\prime}\right) \triangleq \begin{cases}\left(\sigma \cup \sigma^{\prime}, \mu \otimes \mu^{\prime}\right) & : \sigma=\sigma^{\prime} \text { on } \operatorname{dom}(\sigma) \cap \operatorname{dom}\left(\sigma^{\prime}\right) \text { and } \operatorname{dom}(\mu) \cap \operatorname{dom}\left(\mu^{\prime}\right)=\emptyset \\ \text { undefined } & : \text { otherwise. }\end{cases}
$$

Let e be the empty deterministic memory paired with the Dirac distribution over the empty probabilistic memory, and let $\sqsubseteq$ be the following partial order:

$$
(\sigma, \mu) \sqsubseteq\left(\sigma^{\prime}, \mu^{\prime}\right) \quad \text { iff } \quad\left\{\begin{array}{l}
\operatorname{dom}(\sigma) \subseteq \operatorname{dom}\left(\sigma^{\prime}\right) \text { and } \sigma=\sigma^{\prime} \text { on } \operatorname{dom}(\sigma) \\
\operatorname{dom}(\mu) \subseteq \operatorname{dom}\left(\mu^{\prime}\right) \text { and } \mu=\pi_{\operatorname{dom}\left(\mu^{\prime}\right), \operatorname{dom}(\mu)}\left(\mu^{\prime}\right) .
\end{array}\right.
$$


Then $(M, \circ, e, \sqsubseteq)$ is a Kripke resource monoid.

To describe basic properties of configurations, we take the following atomic formulas.

$$
\mathcal{A P} \ni p::=\mathrm{U}_{S}[\mathcal{R E}]|\mathcal{R} \mathcal{E} \sim \mathcal{R} \mathcal{E}| \mathcal{D E}=\mathcal{D} \mathcal{E}|\mathcal{D E} \leq \mathcal{D E}| \cdots
$$

We will fix a base theory $E$ of program expressions, enough to interpret the necessary arithmetic operations $(+, \cdot)$ and relations $(=, \leq)$, and we write $\models_{E} \phi$ if $\phi$ when $\phi$ is valid. For example $\models_{E}$ $e+e^{\prime}=e^{\prime}+e$ holds for any two expressions, randomized or not.

Validity for atomic formulas of deterministic expressions is defined as expected: these formulas can be interpreted as subsets of DetM. More formally, for any deterministic proposition $p_{d}$ we write $\sigma \models_{E} p_{d}$ if $p_{d}$ holds in $\sigma$, and we define:

$$
(\sigma, \mu) \vDash p_{d} \text { iff } \sigma \vDash \models_{E} p_{d}
$$

The more interesting cases are the atomic formulas for randomized expressions.

Definition 4. For a nonempty finite set $S$ and a randomized expression $e_{r} \in \mathcal{R E}$, we define $\llbracket \mathrm{U}_{S}\left[e_{r}\right] \rrbracket$ to be the set of configurations $(\sigma, \mu)$ where $F V\left(e_{r}\right) \subseteq \operatorname{dom}(\sigma) \cup \operatorname{dom}(\mu)$, and $\llbracket e_{r} \rrbracket(\sigma, \mu)$ assigns probability $1 /|S|$ to each element of $S$; we omit $S$ when it is clear from the context.

We define $\llbracket e_{r} \sim e_{r}^{\prime} \rrbracket$ to be the set of configurations $(\sigma, \mu)$ where $F V\left(e_{r}\right) \cup F V\left(e_{r}^{\prime}\right) \subseteq \operatorname{dom}(\sigma) \cup \operatorname{dom}(\mu)$ and $\llbracket e_{r} \rrbracket(\sigma, m)=\llbracket e_{r}^{\prime} \rrbracket(\sigma, m)$ for $m \in \operatorname{supp}(\mu)$. This formula asserts equality between randomized expressions; we use $\sim$ to avoid confusion with equality between deterministic expressions. We abbreviate $\mathrm{D}[e] \triangleq e \sim e$. Explicitly, $\llbracket \mathrm{D}[e] \rrbracket$ contains all configurations $(\sigma, \mu)$ where $F V(e) \subseteq \operatorname{dom}(\sigma) \cup \operatorname{dom}(\mu)$.

Since the interpretation of atomic assertions is monotonic, our configurations are a Kripke resource model of BI. An important feature of the resulting semantics is that validity only depends on the variables in the formula. (We defer proofs to the full version [Barthe et al. 2020].)

Lemma 1 (Restriction). Let $(\sigma, \mu)$ be any configuration and let $\phi$ be a BI formula. Then:

$$
(\sigma, \mu) \vDash \phi \Longleftrightarrow\left(\sigma, \pi_{F V(\phi)}(\mu)\right) \vDash \phi .
$$

One useful consequence is the following property, which allows a $\wedge$ conjunct to be pulled into a * conjunct covering all of the formula's free random variables.

Lemma 2 (Extrusion). If $\vDash \phi \rightarrow \mathrm{D}[F V(\eta) \cap \mathcal{R} \mathcal{V}]$, then $\vDash(\phi * \psi) \wedge \eta \rightarrow(\phi \wedge \eta) * \psi$.

An easy and useful consequence follows when $\eta$ does not mention any random variables. ${ }^{2}$

Corollary 1. Let $p_{d}$ be a deterministic proposition. The following axiom is sound:

$$
\vDash \psi \wedge p_{d} \rightarrow \psi * p_{d}
$$

Proof. Since $\mathrm{T}$ is the unit for $*$ in our semantics, $\psi \wedge p_{d}$ implies $(T * \psi) \wedge p_{d}$. Since $p_{d}$ does not mention any random variables, Lemma 2 implies $\left(T \wedge p_{d}\right) * \psi$. Symmetry of $*$ gives $\psi * p_{d}$.

We briefly mention two other important features of our semantics. First, the semantics is intuitionistic: $\phi \vee \neg \phi$ is not valid. Second, our semantics admits weakening:

$$
\vDash \phi * \psi \rightarrow \phi \wedge \psi
$$

We will use repeatedly this property to pull out facts about specific variables from a larger assertion.

${ }^{2}$ For readers familiar with separation logic, deterministic propositions resemble pure assertions in the heap model of BI. 


\subsection{Axiom Schema for Atomic Formulas}

Next, we present our axioms for atomic formulas. Much like the situation for atomic formulas in the "pointer logic" underlying standard separation logic, these axioms are not complete. Nevertheless, they are already sufficient to reason about many interesting probabilistic programs.

We begin with axioms for formulas with $\sim$. The main difference between $\sim$ and standard equality is that since $\sim$ is interpreted in a randomized configuration-which might not have all of $\mathcal{R} \mathcal{V}$ in its domain-replacing expressions by equal expressions must not introduce new random variables.

Lemma 3. The following axiom schema are valid:

$$
\begin{aligned}
& \vDash e_{r} \sim e_{r}^{\prime} \rightarrow e_{r}^{\prime} \sim e_{r} \\
& \vDash e_{r} \sim e_{r}^{\prime} \wedge e_{r}^{\prime} \sim e_{r}^{\prime \prime} \rightarrow e_{r} \sim e_{r}^{\prime \prime} \\
& \vDash e_{r} \sim e_{r}^{\prime} \rightarrow e_{r} \sim e_{r}^{\prime \prime} \text { whenever } \models_{E} e_{r}^{\prime}=e_{r}^{\prime \prime} \text { and } F V\left(e_{r}^{\prime \prime}\right) \cap \mathcal{R V} \subseteq F V\left(e_{r}^{\prime}\right) \cap \mathcal{R V} \\
& \vDash e_{r} \sim e_{r} \rightarrow e_{r}^{\prime} \sim e_{r}^{\prime} \text { whenever } F V\left(e_{r}^{\prime}\right) \cap \mathcal{R V} \subseteq F V\left(e_{r}\right) \cap \mathcal{R V}
\end{aligned}
$$

Note that $=e_{r} \sim e_{r}$ is not an axiom-it is not sound, since it may not hold in a randomized memory $\mathbf{D}(\operatorname{RanM}[\emptyset])$ with empty domain. We also have axioms for uniformity propositions.

Lemma 4. The following axiom schema are valid:

$$
\begin{aligned}
& \vDash e_{r} \sim e_{r}^{\prime} \wedge \mathbf{U}_{S}\left[e_{r}\right] \rightarrow \mathbf{U}_{S}\left[e_{r}^{\prime}\right] \\
& \vDash \mathbf{U}_{S}\left[e_{r}\right] \rightarrow e_{r} \sim e_{r} \\
& \vDash \mathbf{U}_{S}\left[e_{r}\right] \rightarrow \mathbf{U}_{S}\left[f\left(e_{r}\right)\right] \text { for any bijection } \llbracket f \rrbracket: S \rightarrow S \text { and } F V(f) \cap \mathcal{R V} \subseteq F V\left(e_{r}\right) \cap \mathcal{R V}
\end{aligned}
$$

\subsection{Comparison with Typical Models of BI}

This subsection contains a more detailed comparison with other models of BI; readers who are primarily interested in the separation logic can safely skip ahead to Section 4.

Our model of BI is strongly inspired by the standard heap model. There, worlds are partial maps from heap locations to values and the main atomic assertion $e \mapsto e^{\prime}$ ("points-to") indicates that in the current heap, the location stored in expression $e$ holds the value denoted by $e^{\prime}$ in the current store. A separating conjunction of two points-to assertions $e \mapsto e^{\prime} * f \mapsto f^{\prime}$ indicates that the addresses held in $e$ and $f$ do not alias. This separation property cannot be deduced syntacticallytwo expressions mentioning different variables may refer to the same heap location in the current store-but it is crucial for local reasoning in the presence of aliasing.

Our probabilistic model is designed to capture a fundamentally different notion of separation that is natural to the probabilistic setting. The randomized portion of the program state plays the role of the heap in the heap model, but randomized variables are not heap-allocated. Accordingly, the names of randomized variables are fixed and it is possible to syntactically determine when two assertions refer to the same variable. However, it is not always possible to determine when two assertions refer to probabilistically independent variables-for instance, the assertion $\mathbf{U}[x] \wedge \mathbf{U}[y]$ holds in any memory where $x$ and $y$ are uniform, but $x$ and $y$ may be correlated. The stronger property of probabilistic independence-the notion of non-aliasing that probabilistic BI is designed to model-is captured by the assertion $\mathbf{U}[x] * \mathbf{U}[y]$.

As we have noted, our semantics is intuitionistic. This aspect stems from our choice of a nontrivial partial order $\sqsubseteq$ over worlds. If this order is taken to be discrete, relating only identical elements, and atomic formulas are interpreted exactly, referring to the entire memory rather than a fragment, we would arrive at a classical or Boolean semantics for probabilistic BI. This situation is mirrored in heap models of BI, where the classical logic BBI has a semantics with a discrete order. 


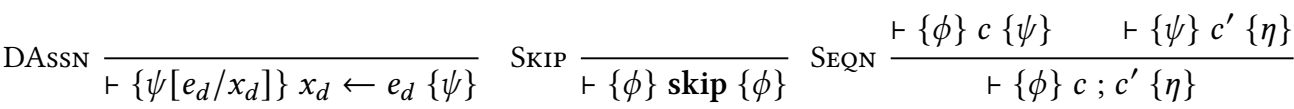

$$
\begin{aligned}
& \vdash\{\phi \wedge b=t t\} c\{\psi\} \\
& \text { DCond } \frac{\vdash\{\phi \wedge b=f f\} c^{\prime}\{\psi\}}{\vdash\{\phi\} \text { if }_{D} b \text { then } c \text { else } c^{\prime}\{\psi\}} \quad \text { DLoop } \frac{\vdash\{\phi \wedge b=t t\} c\{\phi\}}{\vdash\{\phi\} \text { while } b \text { do } c\{\phi \wedge b=f f\}}
\end{aligned}
$$

Fig. 2. Proof rules: deterministic constructs

For heap models, $\mathrm{BBI}$ is more precise than $\mathrm{BI}-$ it supports atomic assertions that are not preserved under heap extension, like emp for empty heap, and BI formulas $\phi$ can be recovered by BBI formulas $\phi * T$. In the probabilistic setting, however, a classical logic seems to run into trouble. For instance, it is not the case that a formula $\phi$ valid in $(\sigma, \mu)$ under our semantics can be directly translated into a classical semantics: $(\sigma, \mu) \vDash \phi *$ T would state that the domain of the distribution modeling $\phi$ is probabilistically independent of all other variables, which does not follow from $(\sigma, \mu) \vDash \phi$. We leave the development of a classical version of probabilistic BI for future work.

\section{A PROBABILISTIC SEPARATION LOGIC}

We now have all the ingredients needed for our separation logic PSL. First, the judgments.

\subsection{Judgments and Validity}

Definition 5. PSL judgments have the form $\{\phi\} c\{\psi\}$ where $\phi$ and $\psi$ are probabilistic BI formulas. Such a judgment is valid, denoted $\models\{\phi\} c\{\psi\}$, if for all configurations $(\sigma, \mu) \in \operatorname{DetM}[\mathcal{D V}] \times$ $\mathbf{D}(\operatorname{RanM}[\mathcal{R} \mathcal{V}])$ satisfying $(\sigma, \mu) \vDash \phi$, we have $\llbracket c \rrbracket(\sigma, \mu) \vDash \psi$.

We have defined validity to quantify over only input states with all variables in the domain. Unlike in separation logic, programs do not allocate variables and memory faults are not possible, so there is no reason to consider behaviors from partial configurations in the program logic.

\subsection{Proof Rules: Deterministic Constructs}

We introduce the proof system of PSL in three stages. First, we consider the deterministic constructs in Fig. 2. The rule DAssn is the usual Hoare rule for assignments, but it is only sound for assignments to deterministic variables. Otherwise, the rules are as expected.

When proving judgments of for-loops, we will use the following derived rule:

$$
\text { DFor } \frac{\vdash\{\phi\} c\{\phi[i+1 / i]\} \quad F V(N) \cap M V(c)=\emptyset}{\vdash\{\phi[1 / i]\} \text { for } i=1, \ldots, N \text { do } c\{\phi[N+1 / i]\}}
$$

$M V(c)$ is the set of variables that may be modified by $c$; we defer the formal definition to Definition 7 , when we discuss the frame rule.

\subsection{Proof Rules: Probabilistic Constructs}

Fig. 3 presents the proof rules for randomized operations. RAsSN and RSAMP are for randomized assignment and random sampling, respectively; in contrast to DAssN, these rules reason forwards. Both rules are presented in their "local" form, where the pre-condition is trivial. We will soon derive "global" variants, with general pre-conditions, from the structural rules.

There are two rules for randomized conditionals. RDCond resembles DCoND, with a sidecondition to ensure that the randomized guard $b$ is deterministic. RCoND applies when the guard 


$$
\begin{gathered}
\operatorname{RAssn} \frac{x_{r} \notin F V\left(e_{r}\right)}{\vdash\{T\} x_{r} \leftarrow e_{r}\left\{x_{r} \sim e_{r}\right\}} \quad \text { RSAMP } \frac{}{\vdash\{T\} x_{r} \leftrightarrow \mathrm{U}_{S}\left\{\mathrm{U}_{S}\left[x_{r}\right]\right\}} \\
\operatorname{RDCOND} \frac{\vdash\{\phi \wedge b \sim t t\} c\{\psi\} \quad \vdash\{\phi \wedge b \sim f f\} c^{\prime}\{\psi\} \quad \vDash \phi \rightarrow(b \sim t t \vee b \sim f f)}{\vdash\{\phi\} \text { if }_{R} b \text { then } c \text { else } c^{\prime}\{\psi\}} \\
\operatorname{RCOND} \frac{\vdash\{\phi * b \sim t t\} c\{\psi * b \sim t t\} \quad \vdash\{\phi * b \sim f f\} c^{\prime}\{\psi * b \sim f f\} \quad \psi \in \mathrm{SP}}{\vdash\{\phi * \mathrm{D}[b]\} \text { if }_{R} b \text { then } c \text { else } c^{\prime}\{\psi * \mathrm{D}[b]\}}
\end{gathered}
$$

Fig. 3. Proof rules: probabilistic constructs

is truly probabilistic, and it has two unusual aspects. First, the pre-condition in the conclusion requires the guard to be separated from the rest of the pre-condition; that is, the guard must be probabilistically independent of the portion of the randomized memory satisfying $\phi$. This separation is crucial for $\phi$ to be soundly used as a pre-condition in each branch: the input distribution to each branch is obtained by conditioning on the value of the guard expression in the input distribution. This operation may not preserve $\phi$, even if $\phi$ and the guard have no variables in common-this is a particular feature of the probabilistic setting.

Example 1. Suppose that $x, y, z$ are randomized boolean variables, and let $\mu$ be the output of:

$$
x \leftrightarrow \mathbf{U}_{\mathbb{B}} ; y \leftrightarrow \mathbf{U}_{\mathbb{B}} ; z \leftarrow x \vee y
$$

In words, $x$ and $y$ store the results of two fair coin flips, and $z$ stores the value of $x \vee y$. Then $x$ and $y$ are independent in $\mu$, i.e., $\mathrm{D}[x] * \mathrm{D}[y]$ holds in $\mu$. However, if $S \subseteq \operatorname{RanM}[\mathcal{R V}]$ is the set of all randomized memories where $z=t t$, representing the event that $z$ is true, then $\mathrm{D}[x] * \mathrm{D}[y]$ does not hold in $\mu \mid S$. Intuitively, if we know $z=t t$, then $x$ and $y$ are correlated: if one is false, then the other must be true.

RCoND also shows that the guard remains independent of the branch post-condition assuming the branches do not modify the guard, and the branch post-condition determines a unique portion of the distribution over randomized memories. Formally, we adapt the following class of assertions from separation logic [Reynolds 2008].

Definition 6. A formula $\phi$ is supported (SP) if for any deterministic memory $\sigma$, there exists a randomized memory $\mu$ such that if $\left(\sigma, \mu^{\prime}\right) \vDash \phi$, then $\mu \sqsubseteq \mu^{\prime}$.

The following syntactic conditions ensure SP.

Lemma 5. The following assertions are SP:

$$
\eta::=p_{d}|\mathcal{R} \mathcal{V} \sim \mathcal{D} \mathcal{E}| \mathrm{U}_{S}[\mathcal{R V}] \mid \eta * \eta
$$

Proof. By induction on $\eta$. The base cases are immediate: $p_{d}$ holds in the unique randomized memory with empty domain, while $x \sim e$ and $\mathrm{U}_{S}[x]$ hold in unique randomized memories with domain $\{x\}$. The inductive case is also straightforward.

Example 2 (Non-SP assertions). A simple example of an assertion that is not covered by Lemma 5 is $\phi \triangleq \mathrm{D}[x]$, where $x \in \mathcal{R} \mathcal{V}$ is a boolean randomized variable. It is easy to see that $\phi$ is not SP; for instance, $\phi$ holds in two incomparable distributions $\delta_{(x \mapsto t t)}$ and $\delta_{(x \mapsto f f)}$. Indeed, allowing $\phi$ as a branch post-condition in RCOND would be unsound. Consider the following program:

$$
c \triangleq \text { if }_{R} b \text { then } x \leftarrow t \text { else } x \leftarrow f f
$$




$$
\begin{aligned}
& \text { WeAK } \frac{\vdash\{\phi\} c\{\psi\} \quad \vDash \phi^{\prime} \rightarrow \phi \wedge \psi \rightarrow \psi^{\prime}}{\vdash\left\{\phi^{\prime}\right\} c\left\{\psi^{\prime}\right\}} \quad \operatorname{TrUE} \frac{}{\vdash\{T\} c\{T\}} \\
& \operatorname{CoNJ} \frac{\vdash\left\{\phi_{1}\right\} c\left\{\psi_{1}\right\} \quad \vdash\left\{\phi_{2}\right\} c\left\{\psi_{2}\right\}}{\vdash\left\{\phi_{1} \wedge \phi_{2}\right\} c\left\{\psi_{1} \wedge \psi_{2}\right\}} \quad \operatorname{CASE} \frac{\vdash\left\{\phi_{1}\right\} c\left\{\psi_{1}\right\} \quad \vdash\left\{\phi_{2}\right\} c\left\{\psi_{2}\right\}}{\vdash\left\{\phi_{1} \vee \phi_{2}\right\} c\left\{\psi_{1} \vee \psi_{2}\right\}} \\
& \operatorname{RCASE} \frac{\vdash\{\phi * b \sim t t\} c\{\psi * b \sim t t\} \quad \vdash\{\phi * b \sim f f\} c\{\psi * b \sim f f\} \quad \psi \in \mathrm{SP}}{\vdash\{\phi * \mathrm{D}[b]\} c\{\psi * \mathrm{D}[b]\}} \\
& \operatorname{CoNsT} \frac{\vdash\{\phi\} c\{\psi\} \quad F V(\eta) \cap M V(c)=\emptyset}{\vdash\{\phi \wedge \eta\} c\{\psi \wedge \eta\}} \\
& \text { FrAME } \frac{\vdash\{\phi\} c\{\psi\} \quad F V(\eta) \cap M V(c)=\emptyset \quad F V(\psi) \subseteq F V(\phi) \cup W V(c) \quad \vDash \phi \rightarrow \mathrm{D}[R V(c)]}{\vdash\{\phi * \eta\} c\{\psi * \eta\}}
\end{aligned}
$$

Fig. 4. Structural rules

Clearly, $\phi * b \sim t t$ and $\phi * b \sim f f$ are sound post-conditions for the two branches. But $c$ is semantically equal to $x \leftarrow b$, and $\mathrm{D}[x] * \mathrm{D}[b]$ is not a sound post-condition.

In the full version [Barthe et al. 2020], we consider a variant of RCond that proves a weaker post-condition, but relaxes the requirement on $\psi$ and allows the branches to modify the guard.

\subsection{Structural Rules}

Fig. 4 collects the final group of rules, the structural rules. Weak, True, ConJ, and Case are standard; RCASE is an analog of RCOND. The last two rules are more interesting. Const is the rule of constancy from Hoare logic, which states that formulas $\eta$ that do not mention any of $c$ 's modified variables $M V(c)$ may be conjoined to the pre- and post-condition. This rule is not sound in standard separation logic-motivating the separating conjunction and the frame rule-but it is sound in PSL: writes cannot invalidate assertions about other variables.

But, the post-condition in CONST does not ensure that $\psi$ and $\eta$ refer to probabilistically independent variables. For this stronger guarantee, we need FrAmE. The side conditions mention several classes of variables. Roughly speaking, $R V(c)$ is the set of variables that $c$ may read from, while $W V(c)$ is the set of variables that $c$ must write to (before possibly reading from). $M V(c)$ is the set of variables that $c$ may write to, so $W V(c)$ is a subset of $M V(c)$.

We can approximate these sets using a simple syntactic condition.

Definition 7. $R V, W V, M V$ are defined as follows:

$$
\begin{gathered}
R V\left(x_{r} \leftarrow e_{r}\right) \triangleq F V\left(e_{r}\right) \quad R V\left(x_{r} \leftrightarrow \mathrm{U}_{S}\right) \triangleq \emptyset \quad R V\left(c ; c^{\prime}\right) \triangleq R V(c) \cup\left(R V\left(c^{\prime}\right) \backslash W V(c)\right) \\
R V\left(\text { if }_{R} b \text { then } c \text { else } c^{\prime}\right) \triangleq F V(b) \cup R V(c) \cup R V\left(c^{\prime}\right) \quad R V(\text { while } b \text { do } c) \triangleq R V(c)
\end{gathered}
$$




$$
\begin{aligned}
& W V\left(x_{r} \leftarrow e_{r}\right) \triangleq\left\{x_{r}\right\} \backslash F V\left(e_{r}\right) \quad W V\left(x_{r} \$ \mathbf{U}_{S}\right) \triangleq\left\{x_{r}\right\} \\
& W V\left(c ; c^{\prime}\right) \triangleq W V(c) \cup\left(W V\left(c^{\prime}\right) \backslash R V(c)\right) \\
& W V\left(\text { if }_{R} b \text { then } c \text { else } c^{\prime}\right) \triangleq\left(W V(c) \cap W V\left(c^{\prime}\right)\right) \backslash F V(b)
\end{aligned}
$$

$$
\begin{array}{cc}
M V\left(x_{r} \leftarrow e\right) \triangleq\left\{x_{r}\right\} \quad M V\left(x_{r} \leftrightarrow \mathrm{U}_{S}\right) \triangleq\left\{x_{r}\right\} & M V\left(c ; c^{\prime}\right) \triangleq M V(c) \cup M V\left(c^{\prime}\right) \\
M V\left(\text { if }_{R} b \text { then } c \text { else } c^{\prime}\right) \triangleq M V(c) \cup M V\left(c^{\prime}\right) & M V(\text { while } b \text { do } c) \triangleq M V(c)
\end{array}
$$

Other analyses are possible, so long as non-modified variables are preserved from input to output, and output modified variables depend only on input read variables.

Lemma 6 (Soundness for $R V, W V, M V)$. Let $\left(\sigma^{\prime}, \mu^{\prime}\right)=\llbracket c \rrbracket(\sigma, \mu)$, and let $S_{r}=R V(c), S_{w}=$ $W V(c), S_{c}=\mathcal{R} \mathcal{V} \backslash M V(c)$. Then:

(1) Variables outside of $M V(c)$ are not modified: $\pi_{S_{c}}\left(\mu^{\prime}\right)=\pi_{S_{c}}(\mu)$.

(2) The sets $S_{r}$ and $S_{w}$ are disjoint.

(3) There exists $F: S_{r} \rightarrow \mathbf{D}(\operatorname{RanM}[M V(c)])$ such that $\mu^{\prime}=\operatorname{bind}\left(\mu, m \mapsto F\left(\pi_{S_{r}}(m)\right) \otimes\right.$ unit $\left.\left(\pi_{S_{c}}(m)\right)\right)$.

Returning to Frame, we consider the side-conditions one by one. The first side-condition is as in Const; the framing condition $\eta$ cannot mention any possibly-modified variables. The second condition states that the post-condition $\psi$ can only mention variables (i) already in the pre-condition $\phi$ or (ii) written by $c$. The last condition states that any portion of the randomized memory satisfying $\phi$ must contain all variables read by $c$. Intuitively, these side-conditions ensure that if the framing condition (i) does not mention modified variables and (ii) is initially independent of all read variables, then it is independent of all variables in $\phi$ as well as all written variables-these variables can only depend on read variables, which were initially all independent from the framing condition.

Example 3. Using CONST, we can derive the following global version of RAsSN:

$$
R A S S N^{*} \frac{x_{r} \notin F V\left(\phi, e_{r}\right)}{\vdash\{\phi\} x_{r} \leftarrow e_{r}\left\{\phi \wedge x_{r} \sim e_{r}\right\}}
$$

The set of modified variables is $\left\{x_{r}\right\}$.

Example 4. Using FRAME, we can derive the following global version of RSAMP:

$$
R S A M P^{*} \frac{x_{r} \notin F V(\phi)}{\vdash\{\phi\} x_{r} \$ \mathbf{U}_{S}\left\{\phi * \mathbf{U}_{S}\left[x_{r}\right]\right\}}
$$

There are no read variables, and the modified and written variables are both $\left\{x_{r}\right\}$.

\subsection{Soundness}

As expected, the proof system is sound.

Theorem 2 (Soundness). If $\vdash\{\phi\} c\{\psi\}$ is derivable, then it is valid: $\models\{\phi\} c\{\psi\}$.

We discuss other meta-theoretical properties in Section 7. 


\section{EXAMPLES: CRYPTOGRAPHIC SECURITY}

We demonstrate our logic by proving security for several cryptographic schemes. As we will see, our logic can express and prove two distinct forms of information-theoretic security properties. For more convenient encoding of the protocols, we will work with an extended language with arrays, which can be indexed or assigned to via $x[i]$, and finite tuples, which can be indexed or assigned to via $x .1, x .2$, etc. To write compact assertions about arrays, our assertions will use big versions of the conjunctions, written $\bigwedge_{i \in p(i)} \phi(i)$ and $*_{i \in p(i)} \phi(i)$ where $i$ is a fresh logical variable and $p$ is deterministic and holds for at most finitely many indices. In some examples we use assignments of the form $x \leftarrow e$ where $x \in F V(e)$. Since our assignment rule RASSN does not apply here, these assignments are short for $x_{f} \leftarrow e ; x \leftarrow x_{f}$, where $x_{f}$ is a fresh temporary variable. We give proof sketches in this section; details can be found in the full version [Barthe et al. 2020].

We will need axioms relating uniformity assertions, pairing, and modular arithmetic, the main arithmetic operation in our examples. In general, axioms are strongly dependent on the equational theory of expressions, and it is not clear how to give a complete axiomatization even for just the modular addition operator; we give axioms schema that are broadly useful for our examples.

Lemma 7. Let $q \geq 2$ be any integer, and let $\left\{x_{i}\right\}$ be any finite set of distinct variables. The following axiom schema are sound.

$$
\begin{aligned}
& \models \mathbf{U}_{S_{1}}\left[x_{1}\right] * \cdots * \mathbf{U}_{S_{n}}\left[x_{n}\right] \leftrightarrow \mathbf{U}_{S_{1} \times \cdots \times S_{n}}\left[\left(x_{1}, \ldots, x_{n}\right)\right] \\
& \vDash \mathbf{U}_{\mathbb{Z}_{q}}\left[x_{1}\right] * \mathbf{D}\left[x_{2}\right] * \cdots * \mathbf{D}\left[x_{n}\right] \wedge x_{0} \sim x_{1}+\cdots+x_{n} \bmod q \rightarrow \mathbf{U}_{\mathbb{Z}_{q}}\left[x_{0}\right] * \mathbf{D}\left[x_{2}\right] * \cdots * \mathbf{D}\left[x_{n}\right]
\end{aligned}
$$

In particular, we will use two derived axioms (writing $\oplus$ for xor, addition modulo 2 ):

$$
\begin{aligned}
& \vDash \mathbf{U}_{\mathbb{Z}_{2}}\left[x_{1}\right] * \mathrm{D}\left[x_{2}\right] \wedge x_{0} \sim x_{1} \oplus x_{2} \rightarrow \mathbf{U}_{\mathbb{Z}_{2}}\left[x_{0}\right] * \mathrm{D}\left[x_{2}\right] \\
& \vDash \mathbf{U}_{\mathbb{Z}_{q}}\left[x_{1}\right] * \mathrm{D}\left[x_{2}\right] * \mathrm{D}\left[x_{3}\right] \wedge x_{0} \sim x_{1}+x_{2}+x_{3} \bmod q \rightarrow \mathbf{U}_{\mathbb{Z}_{q}}\left[x_{0}\right] * \mathrm{D}\left[x_{2}\right] * \mathrm{D}\left[x_{3}\right]
\end{aligned}
$$

These axioms also hold for expressions with at most one free variables.

\subsection{Warming Up: The One-Time Pad}

The one-time pad (OTP) is a simple encryption scheme [Katz and Lindell 2014] enjoying a strong property called perfect secrecy. The OTP is a triple of algorithms (Gen, Enc, Dec) parameterized by $n \in \mathbb{N}$, which determines the key space $\mathcal{K}$, message space $\mathcal{M}$, and ciphertext space $C$, each equal to $\{0,1\}^{n}$ :

- Gen: Select a key $k \in \mathcal{K}$ uniformly at random.

- $\operatorname{Enc}_{k}(m)$ : Given a key $k \in \mathcal{K}$ and message $m \in \mathcal{M}$, output the ciphertext $c=m \oplus k$.

- $\operatorname{Dec}_{k}(c)$ : Given a key $k \in \mathcal{K}$ and ciphertext $c \in \mathcal{C}$, output the message $m=c \oplus k$.

We model Gen and Enc with the following code, where $m$ is a deterministic input variable:

$$
k њ\{0,1\}^{n} ; c \leftarrow m \oplus k
$$

But why is the OTP perfectly secret, and what does that mean in the first place? A natural way to define secrecy is to say that "the ciphertext reveals nothing about the plaintext." We can formalize this intuitive notion in two ways. One variant requires that an observer's view is uniformly distributed for all private inputs.

Definition 8 (Perfect Secrecy as Probabilistic Non-Interference). An encryption scheme $\Pi: \mathcal{M} \rightarrow$ $\mathbf{D}(C)$ is perfectly secret if for every pair of messages $m, m^{\prime} \in \mathcal{M}$ and every ciphertext $c \in C$, we have:

$$
\operatorname{Pr}[\Pi(m)=c]=\operatorname{Pr}\left[\Pi\left(m^{\prime}\right)=c\right] .
$$


This notion can be seen as probabilistic non-interference, a generalization of a standard information flow property to the probabilistic setting. As previously noted, perfect secrecy follows from uniformity: if we can show that the output distribution is uniformly distributed, an observer's view is the same for all private inputs since the uniform distribution is unique. In other words, it suffices to show $\mathbf{U}[c]$ as a post-condition, where $c$ denotes the (probabilistic) output of $\operatorname{Enc}_{k}(m)$.

Another way to define security is to treat the secret input-here, the message-as drawn from a distribution, and then require the observer's view to be probabilistically independent of the input for every distribution on inputs. This formulation captures security through an intuitive reading of independence: the public output reveals no new information about the secret input.

Definition 9 (Perfect Secrecy as Input Independence). Let $\mathcal{M}$ be the message space and let $C$ be the ciphertext space. Regarding an encryption scheme $\Pi: \mathcal{M} \rightarrow \mathrm{D}(C)$ as the map $\tilde{\Pi}: \mathrm{D}(\mathcal{M}) \rightarrow$ $\mathrm{D}(\mathcal{M} \times C)$ that preserves the input distribution, $\Pi$ is perfectly secret if the random variables $M$ and $C$ are independent in $\tilde{\Pi}(\mu)$ for every input distribution $\mu$ over $\mathcal{M}$.

Again, we are done if we can show that the output distribution is independent from the input distribution. In other words, it suffices to show $\mathbf{D}[m] * \mathbf{D}[c]$ as a post-condition, where $m$ denotes the secret input and $c$ denotes the (probabilistic) output of $\operatorname{Enc}_{k}(m)$.

Although it turns out that these two formulations of perfect secrecy are equivalent [Katz and Lindell 2014], the proofs of these properties differ. To demonstrate, we show that the OTP is perfectly secret according to both definitions by establishing $\mathbf{U}[c]$ and $\mathbf{D}[m] * \mathbf{D}[c]$ as post-conditions.

5.1.1 Proof of Uniformity. Starting from the trivial pre-condition $\Phi_{1} \triangleq \top$, we would like to prove the post-condition $\Psi \triangleq \mathbf{U}[c]$. Perfect secrecy then follows from Definition 8 . We start by adjoining the random sample for $k$ according to RSAMP:

$\mathbf{U}[k]$.

Then, by RAssn*, assigning to $c$ gives

$$
\mathrm{U}[k] \wedge c \sim m \oplus k .
$$

The xor axiom (U4) gives the desired post-condition:

$$
\Psi \triangleq \mathrm{U}[c] .
$$

5.1.2 Proof of Input Independence. Starting from the pre-condition $\Phi_{1} \triangleq \mathbf{D}[m]$, we would like to prove the post-condition $\Psi \triangleq \mathbf{D}[m] * \mathbf{U}[c]$, now treating $m$ as a randomized variable; perfect secrecy then follows from Definition 9 . We start by using $\mathrm{RSAMP}^{*}$ to adjoin the sample $k$ :

$$
\mathbf{D}[m] * \mathbf{U}[k] .
$$

By RAssN*, assigning to $c$ then gives

$$
\mathbf{D}[m] * \mathbf{U}[k] \wedge c \sim m \oplus k .
$$

Finally, applying the xor axiom (U4) gives

$$
\mathbf{D}[m] * \mathbf{U}[c],
$$

which implies the desired post-condition:

$$
\Psi \triangleq \mathrm{D}[m] * \mathrm{D}[c] .
$$

We now prove both properties for several other constructions. 


\subsection{Private Information Retrieval}

Private information retrieval (PIR) enables a user to retrieve an item from a database server without the server learning which item was requested [Chor et al. 1995]. For instance, one (highly inefficient) scheme just has the server send the entire database to the user. When multiple copies of the database are held by multiple servers, however, significantly more efficient PIR schemes are possible.

We consider the following single-bit two-server PIR scheme by Chor et al. [1995]. Two noncolluding servers $\mathcal{S}_{0}, \mathcal{S}_{1}$ store the same $N$-bit database $D$. A client $C$ wishes to access the $i$-th bit of $D$, denoted $D[i]$, without either server learning any information about $i$. To achieve this, $C$ first uniformly samples an $N$-bit string $q_{0}$. Then, $C$ sends $q_{0}$ to $\mathcal{S}_{0}$ and sends $q_{1}=q_{0} \oplus I$ to $\mathcal{S}_{1}$, where $I \in\{0,1\}^{N}$ has value 1 at index $i$ and value 0 everywhere else. Server $\mathcal{S}_{0}$ computes a response $r_{0}$ as

$$
\bigoplus_{1 \leq j \leq N: q_{0}[j]=1} D[j] .
$$

Similarly, server $\mathcal{S}_{1}$ computes a response $r_{1}$ as

$$
\bigoplus_{1 \leq j \leq N: q_{1}[j]=1} D[j] .
$$

Finally, $C$ computes $r_{0} \oplus r_{1}=D[i]$. Since it is assumed that $\mathcal{S}_{0}$ and $\mathcal{S}_{1}$ do not collude, the fact that the queries $q_{0}$ and $q_{1}$ are each uniformly distributed ensure that no information about the index $i$ is leaked. The fact that $q_{0}$ and $q_{1}$ are not independent, however, means that the protocol does not ensure secrecy in the presence of collusion-information about $i$ may (and in this case, does) leak out through different correlations between $q_{0}$ and $q_{1}$.

The combined program in Fig. 5 models this protocol (arrays $a_{0}, a_{1}$ initialized with 0 ). We es-

$$
\begin{array}{ll}
1 & q_{0} \leftarrow\{0,1\}^{N} ; \\
2 & q_{1} \leftarrow q_{0} \oplus I ; \\
3 & \text { for } i=1, \ldots, N \text { do } \\
4 & \quad \text { if }_{R} q_{0}[i]=1 \text { then } a_{0}[i] \leftarrow D[i] ; \\
5 & \text { if }_{R} q_{1}[i]=1 \text { then } a_{1}[i] \leftarrow D[i] ; \\
6 & r_{0} \leftarrow \bigoplus_{j \in[1, N]} a_{0}[j] ; \\
7 & r_{1} \leftarrow \bigoplus_{j \in[1, N]} a_{1}[j] ; \\
8 & v \leftarrow r_{0} \oplus r_{1} ;
\end{array}
$$

Fig. 5. Private information retrieval (PIR) scheme tablish security by proving two different properties of the program in our logic: uniformity and input independence. While both properties amount to the same security property, their proofs are different and demonstrate our logic's flexibility.

5.2.1 Proof of Uniformity. Starting from the trivial pre-condition $\Phi_{1} \triangleq \top$, we would like to prove the post-condition

$$
\Psi \triangleq \underbrace{\mathrm{U}\left[q_{0}\right]}_{\mathcal{S}_{0} \text { 's view }} \wedge \underbrace{\mathrm{U}\left[q_{1}\right]}_{\mathcal{S}_{1} \text { 's view }} .
$$

This says that the views of $\mathcal{S}_{0}$ and $\mathcal{S}_{1}\left(q_{0}\right.$ and $q_{1}$, respectively) are uniformly random bitstrings.

By RSAMP, adjoining the sampling for $q_{0}$ (line 1) gives

$$
\mathrm{U}\left[q_{0}\right] \text {. }
$$

Since $I$ is a deterministic variable, we can adjoin $\mathrm{D}[I]$ :

$$
\mathrm{U}\left[q_{0}\right] * \mathbf{D}[I] .
$$

By RAssN ${ }^{*}$, assigning to $q_{1}$ (line 2) gives

$$
\mathrm{U}\left[q_{0}\right] * \mathrm{D}[I] \wedge q_{1} \sim q_{0} \oplus I .
$$


$1 \quad r_{0}, r_{1} \stackrel{\$}{\$}\{0,1\}^{k} ;$

$2 d \stackrel{d}{\$}\{0,1\}$;

$3 \quad$ if $R_{R} d=0$ then $r_{d} \leftarrow r_{0} ; r_{1-d} \leftarrow r_{1}$ else $r_{d} \leftarrow r_{1} ; r_{1-d} \leftarrow r_{0}$;

$4 \quad e \leftarrow c \oplus d$;

$5 \quad$ if $_{R} e=0$ then $f_{0} \leftarrow m_{0} \oplus r_{0} ; f_{1} \leftarrow m_{1} \oplus r_{1}$ else $f_{0} \leftarrow m_{0} \oplus r_{1} ; f_{1} \leftarrow m_{1} \oplus r_{0}$;

$6 \quad$ if $_{D} c=0$ then $m_{c} \leftarrow f_{0} \oplus r_{d} ; f_{1-c} \leftarrow m_{1} \oplus r_{1-d}$ else $m_{c} \leftarrow f_{1} \oplus r_{d} ; f_{1-c} \leftarrow m_{0} \oplus r_{1-d}$;

Fig. 6. Oblivious transfer (OT) scheme

Next, we can pull out $\mathrm{U}\left[q_{0}\right]$ like so:

$$
\mathrm{U}\left[q_{0}\right] \wedge\left(\mathbf{U}\left[q_{0}\right] * \mathrm{D}[I] \wedge q_{1} \sim q_{0} \oplus I\right) .
$$

We then apply the xor axiom (U4) to the right conjunct, giving the desired post-condition:

$$
\Psi \triangleq \mathbf{U}\left[q_{0}\right] \wedge \mathbf{U}\left[q_{1}\right]
$$

Since $q_{0}$ and $q_{1}$ are unmodified in the remainder of the program, we can preserve $\Psi$ through to the end using Const and True.

5.2.2 Proof of Input Independence. Starting from the pre-condition $\Phi_{1} \triangleq \mathbf{D}[I]$, now treating $I$ as a randomized variable, we would like to prove the post-condition

$$
\Psi \triangleq \underbrace{\mathrm{D}[I]}_{\text {Index }} * \underbrace{\mathrm{D}\left[q_{0}\right]}_{\mathcal{S}_{0} \text { 's view }} \wedge \underbrace{\mathrm{D}[I]}_{\text {Index }} * \underbrace{\mathrm{D}\left[q_{1}\right]}_{\mathcal{S}_{1} \text { 's view }} .
$$

This says that the views of $\mathcal{S}_{0}$ and $\mathcal{S}_{1}\left(q_{0}\right.$ and $q_{1}$, respectively) are independent of secret index $I$.

By RSAMP ${ }^{*}$, adjoining the sampling for $q_{0}$ (line 1 ) gives

$$
\mathrm{U}\left[q_{0}\right] * \mathbf{D}[I] \text {. }
$$

By RAssn ${ }^{*}$, assigning to $q_{1}$ (line 2) gives

$$
\mathrm{U}\left[q_{0}\right] * \mathrm{D}[I] \wedge q_{1} \sim q_{0} \oplus I .
$$

Next, we can pull out $\mathrm{D}[I] * \mathrm{U}\left[q_{0}\right]$ like so:

$$
\mathbf{D}[I] * \mathbf{U}\left[q_{0}\right] \wedge\left(\mathbf{U}\left[q_{0}\right] * \mathbf{D}[I] \wedge q_{1} \sim q_{0} \oplus I\right) .
$$

We then apply the xor axiom (U4) to the right conjunct, giving

$$
\mathbf{D}[I] * \mathbf{U}\left[q_{0}\right] \wedge \mathbf{D}[I] * \mathbf{U}\left[q_{1}\right],
$$

which implies the desired post-condition:

$$
\Psi \triangleq \mathrm{D}[I] * \mathrm{D}\left[q_{0}\right] \wedge \mathrm{D}[I] * \mathrm{D}\left[q_{1}\right] .
$$

Since $q_{0}$ and $q_{1}$ are unmodified in the remainder of the program, we can preserve $\Psi$ through to the end using Const and True.

\subsection{Oblivious Transfer}

Oblivious transfer (OT) is a common building block in many cryptographic protocols [Rabin 2005]. It involves two parties: a sender $\mathcal{S}$ holding two secrets $m_{0}, m_{1}$ and a receiver $\mathcal{R}$ holding a choice bit $c \in\{0,1\}$. Through the protocol, $\mathcal{R}$ learns the secret $m_{c}$ but nothing about $m_{1-c}$, while $\mathcal{S}$ learns nothing about $c$. If the setup can be performed by a trusted third party $\mathcal{T}$, the following simple protocol implements OT [Rivest 1999]: 
(1) $\mathcal{T}$ sends $\mathcal{S}$ two random $k$-bit strings $r_{0}, r_{1}$.

(2) $\mathcal{T}$ sends $\mathcal{R}$ a random bit $d$ and the string $r_{d}$.

(3) $\mathcal{R}$ sends $\mathcal{S}$ the value $e=c \oplus d$.

(4) $\mathcal{S}$ sends $\mathcal{R}$ the values $f_{0}=m_{0} \oplus r_{e}$ and $f_{1}=m_{1} \oplus r_{1-e}$.

(5) $\mathcal{R}$ computes $m_{c}=f_{c} \oplus r_{d}$.

As part of the trusted setup in steps 1 and 2, $\mathcal{T}$ essentially hands $\mathcal{S}$ and $\mathcal{R}$ one-time pad keys to encrypt their secrets. $\mathcal{R}$ uses $d$ to encrypt its choice $c$ (step 3), $\mathcal{S}$ uses $r_{0}, r_{1}$ to encrypt its secrets $m_{0}, m_{1}$ using $\mathcal{R}$ 's message to pick which key to use for which message (step 4 ), and finally $\mathcal{R}$ uses $r_{d}$ to decrypt one of the secrets (step 5).

The protocol ensures perfect secrecy for $\mathcal{R}$ 's choice $c$, since $e$ is an encryption of $c$ under the OTP with key $d$, which is kept secret from $\mathcal{S}$. The protocol ensures perfect secrecy for one of $\mathcal{S}$ 's secrets $m_{0}, m_{1}$, since $f_{0}, f_{1}$ are encryptions of $m_{0}, m_{1}$ under the OTP with keys $r_{e}, r_{1-e}$, and the fact that $\mathcal{R}$ is given one of the keys $\left(r_{d}\right)$ means that it can decrypt exactly one of $f_{0}$ or $f_{1}$.

The combined program in Fig. 6 models the OT protocol. Stating security for $\mathcal{S}$ requires a bit of work. We first instrument the program with ghost code, shown in gray. Intuitively, the ghost code computes the encrypted version of the wrong message, i.e., the one that $\mathcal{R}$ did not request-the combined view of $\mathcal{R}$ should then be uniform.

5.3.1 Proof of Uniformity. Starting from the trivial pre-condition $\Phi_{1} \triangleq \top$, we would like to prove the post-condition

$$
\Psi \triangleq \underbrace{\left(\mathbf{U}_{k \times k}\left[\left(r_{0}, r_{1}\right)\right] * \mathbf{U}[e]\right)}_{\mathcal{S}^{\prime} \text { s view }} \wedge \underbrace{\left(\mathbf{U}[d] * \mathbf{U}_{k \times k}\left[\left(r_{d}, f_{1-c}\right)\right]\right)}_{\mathcal{R}^{\prime} \text { ' "view" }} .
$$

To establish $\mathcal{R}^{\prime} s$ secrecy, we need to consider the view of $\mathcal{S}$, which consists of $r_{0}, r_{1}$, and $e$. For $\mathcal{R}$ 's choice $c$ to be kept secret, it is required that $\mathbf{U}_{k \times k}\left[\left(r_{0}, r_{1}\right)\right] * \mathbf{U}[e]$, i.e., $\mathcal{S}$ 's combined view is uniform Note that it is not enough to establish that the individual components of $\mathcal{S}$ 's view are uniform. To see why, suppose $\mathcal{T}$ also sends $\mathcal{S}$ the random bit $d$, which reveals $c=e \oplus d$. Although the individual components of $\mathcal{S}$ 's view would indeed be uniform, i.e., $\mathbf{U}_{k}\left[r_{0}\right] \wedge \mathbf{U}_{k}\left[r_{1}\right] \wedge \mathbf{U}[e] \wedge \mathbf{U}[d]$, $\mathcal{R}$ 's secrecy is clearly violated. The stronger post-condition establishes that $\mathcal{S}$ 's combined view is uniform.

To establish $\mathcal{S}^{\prime} s$ (one-sided) secrecy, we need to consider the view of $\mathcal{R}$, which consists of $d$, $r_{d}$, and one of $f_{0}$ or $f_{1}$. In particular, the $f_{i}$ to be considered corresponds to the encryption of the "wrong" message, which we assign to the ghost variable $f_{1-c}$ (which is, in turn, computed using the ghost variable $\left.r_{1-d}\right)$. Similar to $\mathcal{R}$ 's secrecy, it is then required that $\mathbf{U}[d] * \mathbf{U}_{k \times k}\left[\left(r_{d}, f_{1-c}\right)\right]$, i.e., $\mathcal{R}$ 's combined view is uniform.

We first show $\mathcal{R}$ 's secrecy, followed by $\mathcal{S}$ 's secrecy, and then combine the results using ConJ. By RSAMP and RSAMP*, we can adjoin the random samplings for $r_{0}, r_{1}, d$ (lines 1-2), giving

$$
\mathbf{U}_{k}\left[r_{0}\right] * \mathbf{U}_{k}\left[r_{1}\right] * \mathbf{U}[d] .
$$

Since the free variables of this formula are unmodified in the conditional (line 3), we can preserve the formula using Const and TRUE. Since $c$ is a deterministic variable we can adjoin $\mathrm{D}[c]$, giving

$$
\mathbf{D}[c] * \mathbf{U}_{k}\left[r_{0}\right] * \mathbf{U}_{k}\left[r_{1}\right] * \mathbf{U}[d] .
$$

For the assignment to $e$ (line 4), we start from the local pre-condition

$$
\mathbf{D}[c] * \mathbf{U}[d] .
$$

By RAssN*, assigning to $e$ gives

$$
(\mathrm{D}[c] * \mathrm{U}[d]) \wedge e \sim c \oplus d .
$$


Applying the xor axiom (U4) leaves

$\mathrm{U}[e]$.

Then, we can frame as follows:

$\operatorname{Frame} \frac{\vdash\{\phi\} c^{\prime}\{\psi\} \quad F V(\eta) \cap M V\left(c^{\prime}\right)=\emptyset \quad F V(\psi) \subseteq F V(\phi) \cup W V\left(c^{\prime}\right) \quad \vDash \phi \rightarrow \mathrm{D}\left[R V\left(c^{\prime}\right)\right]}{\vdash\{\underbrace{\mathbf{D}[c] * \mathbf{U}[d]}_{\phi} * \underbrace{\mathbf{U}_{k}\left[r_{0}\right] * \mathbf{U}_{k}\left[r_{1}\right]}_{\eta}\} \underbrace{e \leftarrow c \oplus d}_{c^{\prime}}\{\underbrace{\mathbf{U}[e]}_{\psi} * \underbrace{\mathbf{U}_{k}\left[r_{0}\right] * \mathbf{U}_{k}\left[r_{1}\right]}_{\eta}\}}$

The post-condition implies

$$
\mathbf{U}_{k \times k}\left[\left(r_{0}, r_{1}\right)\right] * \mathbf{U}[e],
$$

which establishes $\mathcal{R}$ 's secrecy. Since $r_{0}, r_{1}$, and $e$ are unmodified in the remainder of the program, we can preserve $\mathbf{U}_{k \times k}\left[\left(r_{0}, r_{1}\right)\right] * \mathbf{U}[e]$ through to the end using Const.

Next, we show $\mathcal{S}$ 's secrecy. Again, by RSAmP and RSAmP*, we can adjoin the random samplings for $r_{0}, r_{1}, d$ (lines 1-2), giving

$$
\mathbf{U}_{k}\left[r_{0}\right] * \mathbf{U}_{k}\left[r_{1}\right] * \mathbf{U}[d] .
$$

We go through the conditional (line 3) with RConD, which gives pre-condition

$$
\mathbf{U}_{k}\left[r_{0}\right] * \mathbf{U}_{k}\left[r_{1}\right] * d=0 \sim t t .
$$

To go through the first assignment, we start from the local pre-condition

$$
\mathbf{U}_{k}\left[r_{0}\right] \text {. }
$$

By RAssN*, assigning to $r_{d}$ gives

$$
\mathbf{U}_{k}\left[r_{0}\right] \wedge r_{d} \sim r_{0} .
$$

Transferring the distribution law gives

$$
\mathbf{U}_{k}\left[r_{d}\right] \text {. }
$$

We can then frame in $\mathrm{U}_{k}\left[r_{1}\right] * d=0 \sim t t$ giving

$$
\mathbf{U}_{k}\left[r_{d}\right] * \mathbf{U}_{k}\left[r_{1}\right] * d=0 \sim t t .
$$

The second assignment follows similarly, this time starting from the local pre-condition

$$
\mathrm{U}_{k}\left[r_{1}\right]
$$

and giving the following post-condition in the true branch

$$
\mathrm{U}_{k}\left[r_{d}\right] * \mathbf{U}_{k}\left[r_{1-d}\right] * d=0 \sim t t .
$$

The false branch yields the same post-condition, which, by RCond, brings us to the post-condition

$$
\mathbf{U}_{k}\left[r_{d}\right] * \mathbf{U}_{k}\left[r_{1-d}\right] * \mathbf{U}[d] .
$$

Since the free variables of this formula are unmodified in lines $4-5$, we can preserve this formula through using Const and True. Next, we go through the deterministic conditional (line 6) using DConD. In the true branch, we start with pre-condition

$$
\mathbf{U}_{k}\left[r_{d}\right] * \mathbf{U}_{k}\left[r_{1-d}\right] * \mathbf{U}[d] \wedge(c=0)=t t .
$$

Dropping the right conjunct, we can adjoin $\mathbf{D}\left[m_{1}\right]$ like so

$$
\mathbf{U}_{k}\left[r_{d}\right] * \mathbf{D}\left[m_{1}\right] * \mathbf{U}_{k}\left[r_{1-d}\right] * \mathbf{U}[d],
$$

since $m_{1}$ is a deterministic variable. We preserve this formula through the first assignment to $m_{c}$ using Const and TRUe, and then go through the second assignment to $f_{1-c}$ starting from the local pre-condition

$$
\mathbf{D}\left[m_{1}\right] * \mathbf{U}_{k}\left[r_{1-d}\right] .
$$


Applying RAssN* and the xor axiom (U4) gives

$$
\mathbf{U}_{k}\left[f_{1-c}\right] \text {. }
$$

Framing in $\mathbf{U}_{k}\left[r_{d}\right] * \mathbf{U}[d]$ gives the following post-condition in the true branch

$$
\mathbf{U}_{k}\left[r_{d}\right] * \mathbf{U}_{k}\left[f_{1-c}\right] * \mathbf{U}[d] .
$$

The false branch yields the same post-condition. Then, we can merge $\mathbf{U}_{k}\left[r_{d}\right] * \mathbf{U}_{k}\left[f_{1-c}\right]$ and rearrange like so

$$
\mathbf{U}[d] * \mathbf{U}_{k \times k}\left[\left(r_{d}, f_{1-c}\right)\right] .
$$

This establishes $\mathcal{S}$ 's secrecy. Combining the formulas establishing $\mathcal{R}$ 's secrecy and $\mathcal{S}$ 's secrecy using ConJ gives the desired post-condition.

5.3.2 Proof Attempt: Input Independence. Again, we can try to prove security via input independence instead of uniformity. Starting from the pre-condition

$$
\Phi_{1} \triangleq \mathrm{D}[c] \wedge \mathrm{D}\left[m_{0}\right] \wedge \mathrm{D}\left[m_{1}\right],
$$

we would like to prove the post-condition

$$
\Psi \triangleq(\underbrace{\mathbf{D}[c]}_{\mathcal{R}^{\prime} \text { s secret choice }} * \underbrace{\left(\mathbf{D}\left[r_{0}\right] \wedge \mathbf{D}\left[r_{1}\right] \wedge \mathbf{D}[e]\right)}_{\mathcal{S}^{\prime} \text { s view }}) \wedge(\underbrace{\mathbf{D}\left[m_{1-c}\right]}_{\mathcal{S}^{\prime} \text { s unselected secret }} * \underbrace{\left(\mathbf{D}[d] \wedge \mathbf{D}\left[r_{d}\right] \wedge \mathbf{D}\left[f_{0}\right] \wedge \mathbf{D}\left[f_{1}\right]\right)}_{\mathcal{R}^{\prime} \text { s view }}) .
$$

To establish $\mathcal{R}^{\prime}$ s secrecy, we need to show that the secret choice $c$ is independent from $\mathcal{S}$ 's view. Similarly, to establish $\mathcal{S}$ 's (one-sided) secrecy, we need to show that the unselected secret, which we assign to the ghost variable $m_{1-c}$ is independent from $\mathcal{R}$ 's view.

However, here we run into difficulties-it does not seem possible to prove this judgment in our logic, and even sketching a proof on paper is not easy. In general, stating and proving perfect security as input independence is trickier when there are multiple parties, like in OT. Investigating how to prove this kind of property is an interesting direction for further work.

\subsection{Multi-Party Computation}

Secure multi-party computation (MPC) allows mutually untrusting parties to jointly compute a function of their private inputs without revealing them [Goldreich et al. 1987; Yao 1986]. The parties agree on a function $f$ and $\quad 4$ then use an MPC protocol to securely com- 5 pute $v=f\left(x_{1}, \ldots, x_{n}\right)$, where $x_{i}$ is party $P_{i}$ 's 6 private input. MPC guarantees that parties 7 learn $v$, and nothing more.

As an example, we consider secure computation of addition, i.e., of the function

$$
\begin{aligned}
& \text { for } i=1, \ldots, 3 \text { do } \\
& r[i] .1 \$ \mathbb{Z}_{p} ; \\
& r[i] .2 \$ \mathbb{Z}_{p} \text {; } \\
& r[i] .3 \leftarrow x[i]-r[i] .1-r[i] .2 \bmod p ; \\
& \text { for } i=1, \ldots, 3 \text { do } \\
& s[i] \leftarrow r[1] . i+r[2] . i+r[3] . i \bmod p ; \\
& v \leftarrow s[1]+s[2]+s[3] \bmod p \text {; }
\end{aligned}
$$

Fig. 7. Three-party secure addition protocol $f\left(x_{1}, \ldots, x_{n}\right)=\sum_{i=1}^{n} x_{i}$ [Cramer et al. 2015]. This simple function turns out to be surprisingly useful, for example, for privately totaling salaries of employees in a company or votes in secure electronic voting. Secure addition can be achieved by the following simple protocol with inputs $x_{i} \in \mathbb{Z}_{p}$, where $p$ is a fixed prime number agreed upon in advance. We describe the three-party case for simplicity, but the protocol easily extends to $n$ parties.

(1) Each $P_{i}$ encodes their input as three secret shares by choosing $r_{i, 1}, r_{i, 2}$ uniformly at random in $\mathbb{Z}_{p}$ and setting $r_{i, 3}=x_{i}-r_{i, 1}-r_{i, 2} \bmod p$.

(2) Each $P_{i}$ sends $r_{i, 2}, r_{i, 3}$ to $P_{1}, r_{i, 1}, r_{i, 3}$ to $P_{2}$, and $r_{i, 1}, r_{i, 2}$ to $P_{3}$. 
(3) Each $P_{j}$ computes the sum $s_{\ell}=r_{1, \ell}+r_{2, \ell}+r_{3, \ell} \bmod p$ for $\ell \neq j$ and sends $s_{\ell}$ to all parties.

(4) All parties compute the result $v=s_{1}+s_{2}+s_{3} \bmod p$.

The end-to-end security of the protocol, i.e., that parties learn no new information beyond the output $v$, is subtle to prove. In a nutshell, the security of the protocol is usually established by simulation [Lindell 2017], a proof technique that is pervasive in cryptography but does not have a clean translation to our logic. The interested reader should see the monograph by Cramer et al. [2015].

The security of secret sharing, however, is expressible in our logic. Informally, the protocol splits each secret into three pieces ("shares"), and the security property ensures that knowing at most two of the three shares reveals no information about the secret. In step (1), each party generates shares of their secret input $x_{i}$ by selecting $r_{i, 1}, r_{i, 2}, r_{i, 3}$ uniformly at random from $\mathbb{Z}_{p}$, subject to the constraint that the shares add up to $x_{i}$. In step (2), each party distributes secret shares in such a way that no other party learns any information about their secret input. For concreteness, consider $P_{2}$ 's view: It knows the values $r_{i, 1}$ and $r_{i, 3}$, and that $x_{i}=r_{i, 1}+r_{i, 2}+r_{i, 3} \bmod p$, but since $r_{i, 2}$ is chosen uniformly from $\mathbb{Z}_{p}$, any value of $x_{i}$ is equally likely. Thus, no information about $x_{i}$ is leaked.

The combined program in Fig. 7 models the secure addition protocol; the secret sharing steps correspond to lines 1-4. Like in our previous examples, we prove the security of secret sharing in two ways: by establishing uniformity and input independence.

5.4.1 Proof of Uniformity. Starting from the trivial pre-condition $\Phi_{1} \triangleq \top$, we would like to prove the post-condition

$$
\Psi \triangleq \bigwedge_{\alpha \in\{2,3\}} \underbrace{\mathrm{U}[(r[\alpha] .2, r[\alpha] .3)]}_{P_{1}{ }^{\prime} \text { s view from } P_{\alpha}} \wedge \bigwedge_{\alpha \in\{1,3\}} \underbrace{\mathrm{U}[(r[\alpha] .1, r[\alpha] .3)]}_{P_{2}{ }^{\prime} \text { 's view from } P_{\alpha}} \wedge \bigwedge_{\alpha \in\{1,2\}} \underbrace{\mathrm{U}[(r[\alpha] .1, r[\alpha] .2)]}_{P_{3}{ }^{\prime} \text { s view from } P_{\alpha}} .
$$

This says that each party's view from the other parties is uniform and independent.

To prove this post-condition, we take the following for-loop invariant:

$$
\underset{\alpha \in[1, i)}{*} \mathrm{U}[(r[\alpha] .1, r[\alpha] .2)] \wedge \mathrm{U}[(r[\alpha] .2, r[\alpha] .3)] \wedge \mathrm{U}[(r[\alpha] .1, r[\alpha] .3)] .
$$

By RSAMP*, adjoining the random samplings for $r[i] .1$ and $r[i] .2$ (lines 2 and 3 ) gives

$$
\mathbf{U}[r[i] .1] * \mathbf{U}[r[i] .2] * \underset{\alpha \in[1, i)}{*} \mathbf{U}[(r[\alpha] .1, r[\alpha] .2)] \wedge \mathbf{U}[(r[\alpha] .2, r[\alpha] .3)] \wedge \mathbf{U}[(r[\alpha] .1, r[\alpha] .3)] .
$$

To go through the assignment to $r[i] .3$ (line 4), we start from the local pre-condition

$$
\mathbf{U}[r[i] .1] * \mathbf{U}[r[i] .2] .
$$

By RAssN*, assigning to $r[i] .3$ gives

$$
\mathrm{U}[r[i] .1] * \mathbf{U}[r[i] .2] \wedge r[i] .3 \sim x[i]-r[i] .1-r[i] .2 \bmod p .
$$

Applying the modular addition axiom (U5) and merging pairwise independent assertions gives

$$
\mathrm{U}[(r[i] .1, r[i] .2)] \wedge \mathrm{U}[(r[i] .2, r[i] .3)] \wedge \mathrm{U}[(r[i] .1, r[i] .3)] .
$$

Framing in the invariant for the earlier iterations establishes the loop invariant in DFor, giving:

$$
\underset{\alpha \in[1,3]}{*} \mathrm{U}[(r[\alpha] .1, r[\alpha] .2)] \wedge \mathrm{U}[(r[\alpha] .2, r[\alpha] .3)] \wedge \mathrm{U}[(r[\alpha] .1, r[\alpha] .3)] .
$$

After rearranging and dropping terms, this formula implies the desired post-condition $\Psi$. Since the free variables of $\Psi$ are unmodified in the remainder of the program, we can preserve it through to the end using Const and True, establishing uniformity. 
5.4.2 Proof of Input Independence. Starting from the pre-condition

$$
\Phi_{1} \triangleq \bigwedge_{\alpha \in[1,3]} \mathrm{D}[x[\alpha]]
$$

we would like to prove the post-condition

$$
\begin{aligned}
\Psi \triangleq \bigwedge_{\alpha \in\{2,3\}} \underbrace{\mathrm{D}[x[\alpha]]}_{P_{\alpha}^{\prime} \text { 's input }} * \underbrace{\mathrm{D}[(r[\alpha] .2, r[\alpha] .3)]}_{P_{1}{ }^{\prime} \text { s view from } P_{\alpha}} \wedge \bigwedge_{\alpha \in\{1,3\}} \underbrace{\mathrm{D}[x[\alpha]]}_{P_{\alpha}{ }^{\prime} \text { 's input }} * \underbrace{\mathrm{D}[(r[\alpha] .1, r[\alpha] .3)]}_{P_{2} \text { 's view from } P_{\alpha}} \wedge \\
\bigwedge_{\alpha \in\{1,2\}} \underbrace{\mathrm{D}[x[\alpha]]}_{P_{\alpha} \text { 's input }} * \underbrace{\mathrm{D}[(r[\alpha] .1, r[\alpha] .2)]}_{P_{3} \text { 's view from } P_{\alpha}} .
\end{aligned}
$$

This says that, for each party, the secret input of each other party is independent from the view they generate. We defer details of this proof to the full version [Barthe et al. 2020].

\subsection{Simple Oblivious RAM}

Consider a core programming language defined by the following syntax:

$$
P::=\epsilon \mid i ; P \quad \text { where } i::=\operatorname{read}(x) \mid \operatorname{write}(x, v)
$$

with $x$ ranging over a set $\mathcal{X}$ of registers and $v$ ranging over integers $\mathbb{Z}$. A simple execution model for this language is random access memory (RAM). Informally, a RAM machine maintains a partial mapping from registers to integers, a program counter that tracks which instruction is to be executed next, and reads and updates the mapping according to the program instructions.

Oblivious RAM (ORAM) [Goldreich 1987; Goldreich and Ostrovsky 1996] is a probabilistic execution model guaranteeing that an adversary who observes the sequence of accessed memory locations-but not their contents-only learns the length of the program. The basic idea of ORAM is to maintain a mapping from logical addresses accessed by the client program to physical addresses where data is stored; this mapping is re-scrambled after each read and write. We consider a simple and idealized variant of ORAM inspired by Chung and Pass [2013], and use our logic to prove its security.

5.5.1 Definition of Simple ORAM. ORAM assumes a memory model split into two parts: an external, insecure bulk memory where accesses are visible to the adversary, and an internal, secure memory with a small number of registers where accesses are not visible to the adversary. In our variant, the internal memory stores a so-called position map, while the external memory is organized into a tree where values can be read and written. We treat the external memory $a$ as a map from addresses to buckets. Addresses are bitstrings of length at most $n$; they are partially ordered by the prefix relation, and are thus structured as a tree. Leaf addresses are bitstrings of length exactly $n$. Each node of the tree stores a bucket a list of triples of the form $(x, v, l)$, where $x \in \mathcal{X}$ is a register, $v \in \mathbb{Z}$ is an integer, and $l \in\{0,1\}^{n}$ is a leaf address. One key invariant of the ORAM scheme is that $(x, v, l)$ will be stored in some bucket along the path from root address $\epsilon$ to leaf address $l$, i.e. in a bucket $a[i]$ at some address $i$ that is a prefix of $l$. The position map $p$ is a mapping from registers $x \in \mathcal{X}$ to leaf addresses; this mapping ensures obliviousness by introducing a level of indirection.

We briefly describe the semantics of the read $(x)$ instruction; Fig. 8 illustrates an example. To retrieve the value of $x$, we read $p[x]$ from the position map and then search through all buckets along the path from $\epsilon$ to $p[x]$ for a triple of the form $(x, u, p[x])$ (Fig. 8 (a)). Once the triple is found, it is removed from its bucket. In order to guarantee obliviousness, it is necessary to read all addresses along the path to the leaf, regardless of where the target entry is found. Once the leaf is reached, we sample a fresh bitstring $l$ of length $n$, add $(x, u, l)$ to the bucket $a[\epsilon]$, and then update 




(a) $\operatorname{read}(x)$

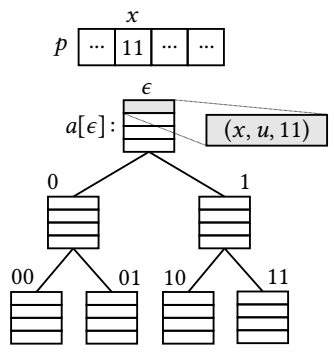

(b) Update $p[x]$

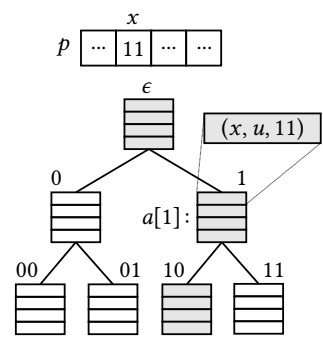

(c) flush

Fig. 8. Simple ORAM

$p[x]$ to hold $l$ (Fig. $8(\mathrm{~b}))$. The semantics of the write $(x, v)$ instruction is similar, except that the updated entry $(x, v, l)$ is added to the bucket $a[\epsilon]$.

While the operations so far ensure obliviousness, all triples will accumulate at the root of the tree, i.e., in the bucket $a[\epsilon]$. To better balance the buckets, each read or write operation is followed by a flush operation, which samples another bitstring $l$ of length $n$ and then traverses the tree from root $\epsilon$ to leaf $l$ while pushing every triple $\left(x, v, l^{\prime}\right)$ along its path as far down as possible, namely, to bucket $a\left[\operatorname{Icp}\left(l, l^{\prime}\right)\right]$ where $\operatorname{Icp}\left(l, l^{\prime}\right)$ is the longest common prefix of $l$ and $l^{\prime}$ (Fig. 8 (c)).

Fig. 9 defines the oblivious semantics of core language programs by compilation to PWHILE. To model the adversary's view of the accesses, the compilation instruments the code to store leakage information in the variable $\ell$, as explained below. We briefly comment on the notation and operators. We use [] for the empty list, :: for adding an element to a list, and + for concatenating two lists Given a bitstring $i$ of length $n$ and $k \leq n$, we let $i[1, \ldots, k]$ be the bitstring of length $k$ consisting of the first $k$ bits of $i$; we let $i[1, \ldots, 0]=\epsilon$ denote the empty bitstring. The operator $\operatorname{split}_{\{(x, v, l) \mid \phi\}}$ iterates over a list of triples and returns two sublists of elements satisfying $\phi$ and elements not satisfying $\phi$ respectively, where $\phi$ can mention $(x, v, l)$.

5.5.2 Security of Simple ORAM. Informally, the leakage of a program is the sequence of internal memory accesses performed during program execution. Formally, we introduce the events $\mathrm{r} a[i]$ and $\mathrm{w} a[i]$ for reading and writing address $i$; note that reading or writing an internal address does not leak any information. Leakage is then defined as a sequence of events. For instance, the leakage for instructions $\operatorname{read}(x)$ or write $(x, v)$ is the sequence:

$$
\mathrm{r} a[\epsilon], \mathrm{w} a[\epsilon], \mathrm{r} a[p[x][1]], \mathrm{w} a[p[x][1]], \ldots, \mathrm{r} a[p[x]], \mathrm{w} a[p[x]], \mathrm{w} a[\epsilon] .
$$

The leakage of a program is the concatenation of the leakage of its instructions. Obliviousness states that executing two different programs with the same number of instructions induce the same leakage, and that the leakage does not depend on the initial contents of the ORAM.

Our proof uses an equivalent definition of obliviousness that is more convenient for our purposes. Concretely, we encode the leakage of instructions read $(x)$ and write $(x, v)$ by $p[x]$. Redefining the leakage in this way does not affect the definition of obliviousness, but the advantage is that we can show obliviousness by proving that the leakage of a program $P$ of length $k$ is uniform over bitstrings of length $2 \cdot n \cdot k .^{3}$ Our compilation adds ghost code to record this leakage in an array $\ell$ indexed by the program counter $c$, which tracks the index of the current instruction. Concretely, we

\footnotetext{
${ }^{3}$ In contrast, using the straightforward definition of leakage would require proving that a block of memory accesses is uniform over paths from root to leaf. Although this can also be done in our logic, the proof becomes more cumbersome.
} 


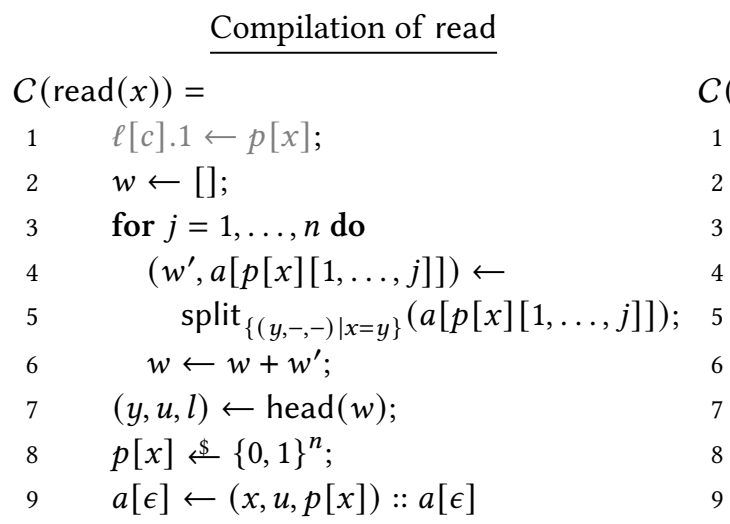

Flush operation

flush $=$

$1 \quad l \$\{0,1\}^{n} ;$

$2 \quad \ell[c] .2 \leftarrow l$;

$3 \quad w \leftarrow[]$;

4 for $j=1, \ldots, n$ do

$5 \quad(a[l[1, \ldots, j]], w) \leftarrow \operatorname{split}_{\left\{\left(-,-, l^{\prime}\right) \mid \operatorname{ccp}\left(l, l^{\prime}\right)=l[1, \ldots, i]\right\}}(a[l[1, \ldots, j]]+w)$

Fig. 9. Compiling programs. Grayed instructions is ghost code used to record leakage.

add an assignment $\ell[c] .1 \leftarrow p[x]$ at the beginning of each instruction (line 1 in both read and write compilation), and an assignment $\ell[c] .2 \leftarrow l$ in corresponding flush operations (line 2 in flush).

Under our encoding, proving security of the ORAM scheme reduces to showing that for every program $P$ of length $k$ in our core language, the leakages $(\ell[1] .1, \ell[1] .2), \ldots,(\ell[k] .1, \ell[k] .2)$ are independently and uniformly distributed in the post-condition of the compiled version of $P$.

We sketch how to formalize this property as a uniformity property in our logic; details are in the full version [Barthe et al. 2020]. The overall strategy is to show that after each instruction-flush pair, the entries of the position map and the leakage are uniform and mutually independent. We establish two judgments

$$
\begin{gathered}
\{\Phi(j)\} C(\operatorname{read}(x)) ; \text { flush ; } c \leftarrow c+1\{\Phi(j+1)\} \\
\{\Phi(j)\} C(\operatorname{write}(x, v)) ; \text { flush } ; c \leftarrow c+1\{\Phi(j+1)\}
\end{gathered}
$$

for every $j$, where the invariant is defined to be

$$
\Phi(j) \triangleq c=j \wedge \underset{\beta \in[1, c)}{*} \mathbf{U}[(\ell[\beta] .1, \ell[\beta] .2)] * \underset{\alpha \in \mathcal{X}}{*} \mathbf{U}[p[\alpha]] .
$$

Starting from the pre-condition $\Phi_{1} \triangleq \Phi(1)$, which asserts that the position map is initialized uniformly and independently, repeatedly applying SEQN establishes

$$
\{\Phi(1)\} C(P)\{\Phi(k+1)\} .
$$

The post-condition implies our desired assertion:

$$
\Psi \triangleq \underset{\beta \in[1, k]}{*} \mathbf{U}[(\ell[\beta] .1, \ell[\beta] .2)],
$$

which says that the distribution of instruction-flush leakage pairs is uniform and independent. 
Discussion. The ORAM scheme by Chung and Pass [2013] has several additional wrinkles. First, they define a recursive ORAM, which uses a hierarchy of trees to reduce the internal memory; this construction can be encoded in our language, and we conjecture that our proof can be extended to this more complex setting. Moreover, Chung and Pass [2013] initialize the position map lazily, i.e. for every instruction write $(x, v)$, the ORAM checks whether the position map for $x$ is already defined, and samples a fresh bitstring and extends the position map otherwise. This lazy version can be modeled using conditionals, and our proof can be adapted to this variant.

Chung and Pass [2013] also assume that buckets have a maximal size. As a consequence, execution may fail if buckets overflow. Formalizing this variant requires care. One option would be to prove that the leakage trace is uniform conditioned on execution not failing, and that the probability of failing is small. However, dealing with conditional uniformity is challenging. A better solution may be to compare the distributions induced by executing the program with finite buckets and with infinite buckets, showing that these distributions are close.

\section{RELATED WORK}

The intersection of programming languages and security is broad; we limit our attention to the most directly relevant work.

Probabilistic independence. Our logic is the first program logic where probabilistic independence is the central concept, but previous systems have also touched on independence. Darais et al. [2020] define a type and effect system for proving properties of probabilistic computations. Their effect system is based on a new notion of probabilistic region, which they use to track probabilistic dependencies. Their type system uses affine typing to ensure that random variables are used at most once. They show the expressiveness of their type and effect system with examples of simple ORAM and tree-based ORAM. Although the two approaches have some similarities, it seems challenging to compare the expressiveness of their type and effect system and of our logic. One advantage of our logic is that it admits an intuitive interpretation based on bunched logics.

Barthe et al. [2009] define probabilistic Relational Hoare Logic (PRHL), a program logic for proving relational specifications of probabilistic programs. Their logic provides a flexible framework for proving information flow properties of programs. These approaches are able to deal with Private Information Retrieval, Multi-Party Computation, but are otherwise incomparable to ours. In particular, it seems difficult to use their approaches for proving security of ORAM in PRHL, without using an additional proof technique called Eager/Lazy Sampling. On the other hand, they can prove that von Neumann's trick, an algorithm to simulate a fair coin using a biased coin, yields a uniform distribution, which appears out of reach of our current proof system. Barthe et al. [2017] also show how to use this logic to prove uniformity and independence for probabilistic programs, but these assertions can only be established at the end of the program.

PSL is also related to ElLORA, a program logic for probabilistic programs [Barthe et al. 2018]. ELLORA works with a more standard assertion logic based on first order logic, and allows assertions to directly describe probabilities of events. This expressivity means that it is possible to reason about independence as a defined assertion. Barthe et al. [2018] propose an "independence logic" as a subsystem, but the rules are limited (e.g., it is not possible to reason about probabilistic control flow). In contrast, probabilistic independence in PSL is handled implicitly by means of a substructural logic. While this kind of logic is a bit exotic, we find that it makes it possible to represent independence assertions more compactly and integrate with mathematical axioms more smoothly.

In more specialized contexts, Smith [2003] and Hoang et al. [2015] develop type systems for proving computational security of modes of operation and authenticated encryption schemes. Their type system enforces a strong invariant probabilistic independence between different expressions, 
although the type system in itself does not feature any specific judgment for probabilistic independence. It would be very interesting to understand whether their results could be emulated and generalized in our program logic. In a similar spirit, Barthe et al. [2015] develop a proof system for proving that programs are protected against power side-channels. Their proof system makes an implicit but critical use of independence.

On the more foundational side, Ackerman et al. [2019] study computability issues for (conditional) independence. Their work is partially motivated by exchangeable sequences, which are closely related to independence. Language-based investigations of exchangeable sequences can be found for instance in Staton et al. [2018]. It would be interesting to investigate how to reason about exchangeable sequences in our logic.

Separation logics for probabilistic programs. There have been two recent proposals for probabilistic separation logics. Batz et al. [2019] developed a logic QSL for reasoning about probabilistic, heapmanipulating programs. There, the connectives in BI are interpreted as acting on expectations, real-valued analogs of state predicates. Tassarotti and Harper [2019] have also developed a relational separation program logic for reasoning about concurrent probabilistic programs. Both of these logics leverage standard notions of separation, from heap separation logic and concurrent separation logic, respectively.

BI and separation logics. Our work builds on fruitful lines of research on bunched implications and separation logic. On the bunched implications side, our model uses the resource interpretation of BI [Pym et al. 2004]; readers should consult Pym [2002] or Docherty [2019] for more information. The idea of using separation to model probabilistic independence has been considered before [Lozes 2010], ${ }^{4}$ but we are not aware of concrete results in this area. From a different point of view, Simpson [2018] develops categorical structures for independence and conditional independence, encompassing independence in heaps, nominal sets, and probability distributions. On the separation logic side, by varying the notion of separation our work is another instance of separation logic, alongside heap separation logic [Ishtiaq and O'Hearn 2001; O'Hearn et al. 2001] and concurrent separation logic [Brookes 2007; O’Hearn 2007]. These areas are too vast to survey here; the draft notes by Reynolds [2008] are a good place to start.

\section{CONCLUSION AND FUTURE DIRECTIONS}

We have presented a novel separation logic for probabilistic programs, using a probabilistic variant of the logic BI where separation models probabilistic independence. Proofs in the separation logic reason in terms of higher-level properties like independence and uniformity, and we have demonstrated our logic to prove two forms of cryptographic security for a number of interesting protocols. We see many possible directions for interesting future work.

Completeness and decidability. Our logic is not complete: there are semantically valid judgments that are not provable from our proof rules. There are several sources of incompleteness. First, the proof rules for randomized conditionals is incomplete: it only allows parts of the pre-condition that are independent of all variables in the guard expression to be carried into the branches, while a finer analysis could allow more general pre-conditions to be preserved or modified in a controlled way. Another source of incompleteness is the interplay between uniformity, independence, and the equational theory of the expression language; even with just the xor operator, it is not clear how to give a complete axiomatization.

On the positive side, our logic may be relatively complete under the following provisos: expressions are variables (i.e., the expression language has no operators); programs are straightline code

${ }^{4}$ Peter O’Hearn and David Pym, personal communication. 
(i.e., sequences of assignments); and assertions are regular or separating conjunctions of uniformity and equality predicates. In this specific case, it could be possible to reflect a complete dependency analysis into the program logic; since entailment between our restricted set of assertions is decidable, this fragment of the logic may also be decidable.

Decidability of entailment for larger classes of assertions is also an interesting open problem. The formulas in our logic bear a superficial resemblance to the "pointer logic" underlying heap separation logic. Decidability for some restricted fragments follows from a small model property [Yang 2001]. Unlike heap models of BI, our probabilistic model works with a fixed collection of locations; however, our setting has probabilistic correlations. We conjecture that fragments of our logic may also enjoy a small model property, perhaps by tracking which subsets of variables are mutually independent.

Enriching the assertion logic. The assertion logic we have presented is based on intuitionistic, propositional BI. We have found this logic to be convenient to work with, but other choices are certainly possible. One natural alternative is to work with a classical logic instead of an intuitionistic one; in standard separation logic, a classical logic supports a useful, backwards style of reasoning through the "magic wand" connective. In the probabilistic setting, a classical logic runs into trouble because probabilistic separation seems too strong-we cannot freely assume that a variable is independent of the rest of the random variables. However, it may be possible to weaken the notion of separation to allow randomness to be shared in tightly controlled ways; we are currently investigating a non-commutative version of BI for this purpose.

There are also natural extensions to our intuitionistic logic. Developing a probabilistic model of predicate BI [Pym 1999] would allow substantially richer assertions. In particular, the lack of existential quantifiers in our logic complicates our proofs and seems to be an obstacle to defining a strongest post-condition calculus. Extending the logic to support reasoning about conditioning would also help make the proof rules more precise.

Supporting quantitative reasoning. While the assertions in our logic describe probability distributions, our logic notably does not support quantitative reasoning: it is not possible to describe the probability of an event, or the expected value of a function. This stands in sharp contrast to other deductive techniques for probabilistic programs, such as PPDL [Kozen 1985] and PGCL [Morgan et al. 1996]. Incorporating some of these tools for reasoning about numeric probabilities could extend the reach of our logic. One possibility is to make an approximate version of the logic with judgments of the form $\vdash_{\epsilon}\{\phi\} c\{\psi\}$, stating that the output distribution is at distance at most $\epsilon$ of a distribution satisfying $\psi$. Such a logic could be obtained by combining ideas of PSL with the union bound logic of Barthe et al. [2016], and could be used to reason about more advanced versions of our examples and further examples from the cryptographic literature (e.g., the PRF/PRP Switching Lemma of Impagliazzo and Rudich [1988]).

Modeling more advanced properties and cryptographic constructions. We have focused on basic, information-theoretic security properties from cryptography in this paper. It would be interesting to understand whether our logic can be used to capture other properties (e.g., active security). Similarly, it would be interesting to explore potential applications of our logic to other constructions, including more complex variants of the constructions we have considered, e.g., Tree ORAM [Gentry et al. 2013; Shi et al. 2011], Path ORAM [Stefanov et al. 2013], Multi-Server ORAM [Chan et al. 2018] as well as other constructions, such as history independent data structures [Micciancio 1997; Naor and Teague 2001; Wang et al. 2014]. More speculatively, it would be interesting to understand whether our logic could be used for reasoning about computational security, or approximate notions of independence and uniformity. 


\section{ACKNOWLEDGMENTS}

We thank the anonymous reviewers and our shepherd Ohad Kammar for their close reading and useful suggestions. The present work was sparked during a workshop at McGill University's Bellairs Research Institute. This work was also partially supported by Office of Naval Research under projects N00014-12-1-0914, N00014-15-1-2750, and N00014-19-1-2292, the University of Wisconsin, a Facebook TAV grant, an NSF Graduate Research Fellowship, and the Max Planck Institute for Software-Systems for hosting some of the authors.

\section{REFERENCES}

Nathanael L. Ackerman, Jeremy Avigad, Cameron E. Freer, Daniel M. Roy, and Jason M. Rute. 2019. On the computability of graphons. In IEEE Symposium on Logic in Computer Science (LICS), Vancouver, British Columbia.

Gilles Barthe, Sonia Belaïd, François Dupressoir, Pierre-Alain Fouque, Benjamin Grégoire, and Pierre-Yves Strub. 2015. Verified Proofs of Higher-Order Masking. In IACR International Conference on the Theory and Applications of Cryptographic Techniques (EUROCRYPT), Sofia, Bulgaria (Lecture Notes in Computer Science), Vol. 9056. Springer-Verlag, 457-485. https://doi.org/10.1007/978-3-662-46800-5_18

Gilles Barthe, Thomas Espitau, Marco Gaboardi, Benjamin Grégoire, Justin Hsu, and Pierre-Yves Strub. 2018. An AssertionBased Program Logic for Probabilistic Programs. In European Symposium on Programming (ESOP), Thessaloniki, Greece. arXiv:cs.LO/1803.05535 https://arxiv.org/abs/1803.05535

Gilles Barthe, Thomas Espitau, Benjamin Grégoire, Justin Hsu, and Pierre-Yves Strub. 2017. Proving uniformity and independence by self-composition and coupling. In International Conference on Logic for Programming, Artificial Intelligence and Reasoning (LPAR), Maun, Botswana (EPiC Series in Computing), Vol. 46. 385-403. http://www.easychair.org/ publications/paper/340344

Gilles Barthe, Marco Gaboardi, Benjamin Grégoire, Justin Hsu, and Pierre-Yves Strub. 2016. A Program Logic for Union Bounds. In International Colloquium on Automata, Languages and Programming (ICALP), Rome, Italy (Leibniz International Proceedings in Informatics), Vol. 55. Schloss Dagstuhl-Leibniz Center for Informatics, 107:1-107:15. https://doi.org/10. 4230/LIPIcs.ICALP.2016.107 arXiv:cs.LO/1602.05681

Gilles Barthe, Benjamin Grégoire, and Santiago Zanella Béguelin. 2009. Formal certification of code-based cryptographic proofs. In ACM SIGPLAN-SIGACT Symposium on Principles of Programming Languages (POPL), Savannah, Georgia. 90-101. https://doi.org/10.1145/1480881.1480894

Gilles Barthe, Justin Hsu, and Kevin Liao. 2020. A Probabilistic Separation Logic. Proceedings of the ACM on Programming Languages 4, POPL (Jan. 2020). arXiv:cs.PL/1907.10708 https://arxiv.org/abs/1907.10708

Kevin Batz, Benjamin Lucien Kaminski, Joost-Pieter Katoen, Christoph Matheja, and Thomas Noll. 2019. Quantitative separation logic: a logic for reasoning about probabilistic pointer programs. Proceedings of the ACM on Programming Languages 3, POPL (2019), 34:1-34:29. https://doi.org/10.1145/3290347

Stephen Brookes. 2007. A semantics for concurrent separation logic. Theoretical Computer Science 375, 1-3 (2007), $227-270$. https://doi.org/10.1016/j.tcs.2006.12.034

T.-H. Hubert Chan, Jonathan Katz, Kartik Nayak, Antigoni Polychroniadou, and Elaine Shi. 2018. More is Less: Perfectly Secure Oblivious Algorithms in the Multi-server Setting. In International Conference on the Theory and Application of Cryptology and Information Security (ASIACRYPT), Brisbane, Australia (Lecture Notes in Computer Science), Thomas Peyrin and Steven D. Galbraith (Eds.), Vol. 11274. Springer-Verlag, 158-188. https://doi.org/10.1007/978-3-030-03332-3_7

Benny Chor, Oded Goldreich, Eyal Kushilevitz, and Madhu Sudan. 1995. Private information retrieval. In IEEE Symposium on Foundations of Computer Science (FOCS), Milwaukee, Wisconsin. 41-50.

Kai-Min Chung and Rafael Pass. 2013. A Simple ORAM. IACR Cryptology ePrint Archive 2013 (2013), 243. http://eprint.iacr. org/2013/243

Ronald Cramer, Ivan Bjerre Damgård, and Jesper Buus Nielsen. 2015. Secure Multiparty Computation. Cambridge University Press.

David Darais, Chang Liu, Ian Sweet, and Michael Hicks. 2020. A Language for Probabilistically Oblivious Computation. Proceedings of the ACM on Programming Languages 4, POPL (Jan. 2020). http://arxiv.org/abs/1711.09305

Simon Docherty. 2019. Bunched Logics: A Uniform Approach. Ph.D. Dissertation. University College London.

Thomas Ehrhard, Michele Pagani, and Christine Tasson. 2018. Measurable cones and stable, measurable functions: a model for probabilistic higher-order programming. Proceedings of the ACM on Programming Languages 2, POPL (2018), 59:1-59:28. https://doi.org/10.1145/3158147

Didier Galmiche, Daniel Méry, and David J. Pym. 2005. The semantics of BI and resource tableaux. Mathematical Structures in Computer Science 15, 6 (2005), 1033-1088. https://doi.org/10.1017/S0960129505004858 
Craig Gentry, Kenny A. Goldman, Shai Halevi, Charanjit S. Jutla, Mariana Raykova, and Daniel Wichs. 2013. Optimizing ORAM and Using It Efficiently for Secure Computation. In International Symposium on Privacy Enhancing Technologies (PETS), Bloomington, Indiana (Lecture Notes in Computer Science), Vol. 7981. Springer-Verlag, 1-18. https://doi.org/10. 1007/978-3-642-39077-7_1

Michèle Giry. 1982. A categorical approach to probability theory. In Categorical Aspects of Topology and Analysis. Springer Berlin Heidelberg, Berlin, Heidelberg, 68-85.

Oded Goldreich. 1987. Towards a Theory of Software Protection and Simulation by Oblivious RAMs. In ACM SIGACT Symposium on Theory of Computing (STOC), New York, New York, Alfred V. Aho (Ed.). 182-194. https://doi.org/10.1145/ 28395.28416

Oded Goldreich, Silvio Micali, and Avi Wigderson. 1987. How to play any mental game. In ACM SIGACT Symposium on Theory of Computing (STOC), New York, New York. 218-229.

Oded Goldreich and Rafail Ostrovsky. 1996. Software Protection and Simulation on Oblivious RAMs. Fournal of the ACM 43 , 3 (1996), 431-473. https://doi.org/10.1145/233551.233553

Viet Tung Hoang, Jonathan Katz, and Alex J. Malozemoff. 2015. Automated Analysis and Synthesis of Authenticated Encryption Schemes. IACR Cryptology ePrint Archive 2015 (2015), 624. http://eprint.iacr.org/2015/624

Russell Impagliazzo and Steven Rudich. 1988. Limits on the Provable Consequences of One-way Permutations. In IACR International Cryptology Conference (CRYPTO), Santa Barbara, California (Lecture Notes in Computer Science), Vol. 403. Springer, 8-26. https://doi.org/10.1007/0-387-34799-2_2

Samin S. Ishtiaq and Peter W. O'Hearn. 2001. BI as an assertion language for mutable data structures. In ACM SIGPLANSIGACT Symposium on Principles of Programming Languages (POPL), London, England. 14-26. https://dl.acm.org/citation. cfm?id=375719

Jonathan Katz and Yehuda Lindell. 2014. Introduction to Modern Cryptography. Chapman and Hall/CRC.

Dexter Kozen. 1981. Semantics of Probabilistic Programs. f. Comput. System Sci. 22, 3 (1981), 328-350. https://doi.org/10. 1016/0022-0000(81)90036-2

Dexter Kozen. 1985. A Probabilistic PDL. f. Comput. System Sci. 30, 2 (1985). https://doi.org/10.1016/0022-0000(85)90012-1

Yehuda Lindell. 2017. How to simulate it-a tutorial on the simulation proof technique. In Tutorials on the Foundations of Cryptography. Springer-Verlag, 277-346.

Étienne Lozes. 2010. Towards information as resource in separation logic. Draft presented at FCS-PrivMod, Edinburgh.

Daniele Micciancio. 1997. Oblivious Data Structures: Applications to Cryptography. In ACM SIGACT Symposium on Theory of Computing (STOC), El Paso, Texas. 456-464. https://doi.org/10.1145/258533.258638

Carroll Morgan, Annabelle McIver, and Karen Seidel. 1996. Probabilistic Predicate Transformers. ACM Transactions on Programming Languages and Systems 18, 3 (1996), 325-353. https://doi.org/10.1145/229542.229547

Moni Naor and Vanessa Teague. 2001. Anti-presistence: history independent data structures. In Proceedings on 33rd Annual ACM Symposium on Theory of Computing, fuly 6-8, 2001, Heraklion, Crete, Greece. ACM, 492-501. https: //doi.org/10.1145/380752.380844

Peter W. O’Hearn. 2007. Resources, concurrency, and local reasoning. Theoretical Computer Science 375, 1-3 (2007), $271-307$. https://doi.org/10.1016/j.tcs.2006.12.035

Peter W. O’Hearn and David J. Pym. 1999. The logic of bunched implications. Bulletin of Symbolic Logic 5, 2 (1999), $215-244$. http://www.math.ucla.edu/\%7Easl/bsl/0502/0502-003.ps

Peter W. O’Hearn, John C. Reynolds, and Hongseok Yang. 2001. Local Reasoning about Programs that Alter Data Structures. In International Workshop on Computer Science Logic (CSL), Paris, France (Lecture Notes in Computer Science), Vol. 2142. Springer-Verlag, 1-19. https://doi.org/10.1007/3-540-44802-0_1

David J. Pym. 1999. On Bunched Predicate Logic. In IEEE Symposium on Logic in Computer Science (LICS), Trento, Italy. 183-192. https://doi.org/10.1109/LICS.1999.782614

D. J. Pym. 2002. The Semantics and Proof Theory of the Logic of Bunched Implications. Applied Logic Series, Vol. 26. Kluwer Academic Publishers. Errata and Remarks maintained at: http://www.cantab.net/users/david.pym/BI-monographerrata.pdf.

David J. Pym, Peter W. O’Hearn, and Hongseok Yang. 2004. Possible worlds and resources: the semantics of BI. Theoretical Computer Science 315, 1 (2004), 257-305. https://doi.org/10.1016/j.tcs.2003.11.020

Michael O. Rabin. 2005. How To Exchange Secrets with Oblivious Transfer. IACR Cryptology ePrint Archive 2005 (2005), 187.

John C. Reynolds. 2008. An Introduction to Separation Logic (Preliminary Draft). Technical Report. ITU University, Copenhagen. https://www.cs.cmu.edu/ jcr/copenhagen08.pdf

Ronald Rivest. 1999. Unconditionally secure commitment and oblivious transfer schemes using private channels and a trusted initializer. (1999).

N. Saheb-Djahromi. 1980. CPO's of Measures for Nondeterminism. Theoretical Computer Science 12 (1980), 19-37. https: //doi.org/10.1016/0304-3975(80)90003-1 
Elaine Shi, T.-H. Hubert Chan, Emil Stefanov, and Mingfei Li. 2011. Oblivious RAM with $O\left((\log N)^{3}\right)$ Worst-Case Cost. In International Conference on the Theory and Application of Cryptology and Information Security (ASIACRYPT), Seoul, South Korea (Lecture Notes in Computer Science), Vol. 7073. Springer, 197-214. https://doi.org/10.1007/978-3-642-25385-0_11

Alex Simpson. 2018. Category-theoretic Structure for Independence and Conditional Independence. Electronic Notes in Theoretical Computer Science 336 (2018), 281-297. https://doi.org/10.1016/j.entcs.2018.03.028

Geoffrey Smith. 2003. Probabilistic Noninterference through Weak Probabilistic Bisimulation. In IEEE Computer Security Foundations Workshop (CSFW), Pacific Grove, California. 3-13. https://doi.org/10.1109/CSFW.2003.1212701

Sam Staton, Dario Stein, Hongseok Yang, Nathanael L. Ackerman, Cameron E. Freer, and Daniel M. Roy. 2018. The BetaBernoulli process and algebraic effects. In International Colloquium on Automata, Languages and Programming (ICALP), Prague, Czech Republic (Leibniz International Proceedings in Informatics), Vol. 107. Schloss Dagstuhl-Leibniz Center for Informatics, 141:1-141:15. https://doi.org/10.4230/LIPIcs.ICALP.2018.141

Emil Stefanov, Marten van Dijk, Elaine Shi, Christopher W. Fletcher, Ling Ren, Xiangyao Yu, and Srinivas Devadas. 2013. Path ORAM: an extremely simple oblivious RAM protocol. In ACM SIGSAC Conference on Computer and Communications Security (CCS), Berlin, Germany. 299-310. https://doi.org/10.1145/2508859.2516660

Joseph Tassarotti and Robert Harper. 2019. A separation logic for concurrent randomized programs. Proceedings of the ACM on Programming Languages 3, POPL (2019), 64:1-64:30. https://doi.org/10.1145/3290377

Matthijs Vákár, Ohad Kammar, and Sam Staton. 2019. A domain theory for statistical probabilistic programming. Proceedings of the ACM on Programming Languages 3, POPL (2019), 36:1-36:29. https://dl.acm.org/citation.cfm?id=3290349

Xiao Shaun Wang, Kartik Nayak, Chang Liu, T.-H. Hubert Chan, Elaine Shi, Emil Stefanov, and Yan Huang. 2014. Oblivious Data Structures. In ACM SIGSAC Conference on Computer and Communications Security (CCS), Scottsdale, Arizona. 215-226. https://doi.org/10.1145/2660267.2660314

Hongseok Yang. 2001. Local Reasoning for Stateful Programs. Ph.D. Dissertation. Champaign, IL, USA. AAI3023240.

Andrew Chi-Chih Yao. 1986. How to generate and exchange secrets. In IEEE Symposium on Foundations of Computer Science (FOCS), Toronto, Ontario. 162-167. 